\title{
Quartärgliederung und Aufbau von Warmzeit-Kaltzeit-Zyklen in Bereichen mit Dominanz periglazialer Hangsedimente, dargestellt am Quartärprofil Dreihausen/Hessen
}

\author{
Udo Sabelberg, Georgeta Mavrocordat, Heinrich Rohdenburg \\ \& ERNST SCHÖNHALS *)
}

Quaternary stratigraphy, paleoecology, climatic cycle, paleosols, periglacial deposits Central Europe

Kurzfassung: Das Quartärprofil Dreihausen/Hessen ist durch Verzahnung von Löß bzw. Lößlehm und periglazialen Hangsedimenten gekennzeichnet. Der über $12 \mathrm{~m}$ hohe Aufschluß wurde geomorphologisch, stratigraphisch und mikromorphologisch untersucht. Es können 13 Bodenbildungs-Sedimentations-Zyklen unterschieden werden. Aufgrund der erzielten Ergebnisse wird das Idealbild eines quartären Warmzeit-Kaltzeit-Zyklus entworfen.

[Quaternary Stratigraphy and the Layering of Interglacial and Glacial Cycles in Areas with a Preponderance of Periglacial Slope-Sediments, Illustrated by the Quaternary Profile of Dreihausen/Hesse]

A bstract: The Quaternary Profile of Dreihausen/Hesse is marked by the dovetailing of loess or loess-loam and periglacial slope-sediments. A geomorphological, stratigraphic and micromorphological study was carried out on an escarpment some 12 metres high. Thirteen different sedimentation-soil development-cycles could be traced. On the basis of the results arrived at it has been possible to construct a model of an ideal quaternary interglacial/glacial cycle.

\section{Vorbemerkungen}

Das Quartärprofil im Basaltbruch Dreihausen (Abb.1) war bereits früher in Teilen (Rohdenburg 1968) oder übersichtsmäßig (Sabelberg, Rohdenburg \& Havelberg 1974) beschrieben worden. Seitdem ist es geomorphologisch, pedologisch und stratigraphisch eingehend untersucht worden ${ }^{1}$ ). Im folgenden werden die wesentlichen Ergebnisse dieser Untersuchungen mitgeteilt.

Entscheidender Anlaß für die detaillierte Untersuchung des Profils war sein ungewöhnlich komplexer und reichhaltiger Aufbau (Abb. 2 u. 3). Schon die ersten Übersichtsaufnahmen im Gelände ließen vermuten, daß in dem Profil eine sehr große Zahl von Interglazialen und Kaltzeiten repräsentiert ist, mit 13 interglazialen Bodenkomplexen (BK 1BK 13) zumindest wesentlich mehr, als es auch heute noch verbreiteten Vorstellungen über die Gliederung des Quartärs entspricht. Ein wichtiges Anliegen war es, durch die Untersuchungen zu klären, inwieweit diese ersten Befunde bestätigt werden können. Es sei vorweg betont, daß die Annahme einer so großen Zahl von Warmzeit-Kaltzeit-Zyklen innerhalb des Quartärs heute nicht mehr als außergewöhnlich angesehen wird, denn sie sind doch inzwischen durch die voneinander unabhängigen Ergebnisse mehrerer Disziplinen,

*) Anschriften der Verfasser: Dr. U. S a b e 1 b e r g, Jasperallee 29, 3300 Braunschweig; Georgeta M a v r o c o rd a t, Institut für Bodenkunde und Bodenerhaltung, Ludwigstr. 23, 6300 LahnGießen 1; Prof. Dr. H. R o h d e n bu r g, Geographisches Institut der T.U. Braunschweig, Lehrstuhl B für Geographie, 3300 Braunschweig; Prof. Dr. E. S c h ön h a ls, Institut für Bodenkunde und Bodenerhaltung, Ludwigstraße 23, 6300 Lahn-Gießen 1.

1) Die Geländearbeiten, insbesondere auch die Entnahme von Kastenproben als Belegmaterial, konnten im Sommer 1976 abgeschlossen werden, bevor das Profil infolge des fortschreitenden Abbaues teilweise zerstört wurde. 
so z. B. durch die Quartärstratigraphie (Kukla 1969, Semmel 1974) und die Paläobotanik (Frenzel 1973, Menke \& Behre 1973) gestützt. Solche Ergebnisse liegen nicht nur aus dem zentraleuropäischen Raum vor, sondern in gleichem Umfang auch aus dem Mediterrangebiet (RohDENBURG \& SAbelberg 1973).

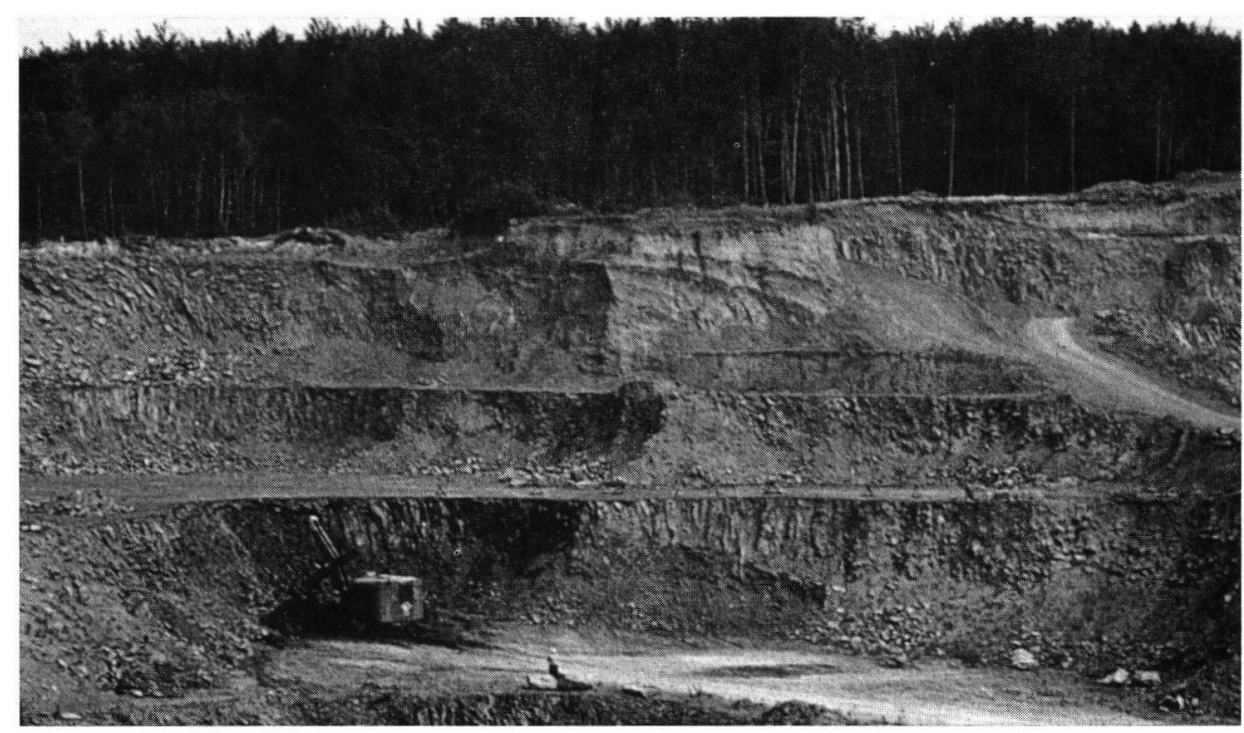

Abb. 1: Basaltbruch der Fa. Nickel, Dreihausen, Gemeinde Ebsdorfer Grund, Kreis Marburg. Blick auf die nördliche Abbauwand, ca. $300 \mathrm{~m} \mathrm{NN}$. In der Mitte des Bildes ist zwischen der Oberfläche und der obersten Abbausohle der Rest der quartären Hohlraumfüllung zu erkennen. Eine zweite, geringmächtige ist oben rechts im Bild zu beobachten.

Neben der die Gliederung des Quartärs insgesamt betreffenden Fragestellung wurde ein Hauptaugenmerk darauf gerichtet, zu bestimmen, wie die einzelnen Warmzeit-Kaltzeit-Zyklen im Detail aufgebaut sind und wie ihre Stellung zueinander hinsichtlich der Vergleichbarkeit ihres Aufbaues zu werten ist. Aus der Klärung dieser Probleme konnten weitere Argumente für oder gegen die getroffene generelle quartärstratigraphische Ansprache des Profils erwartet werden. Wegen des außerordentlich komplizierten Aufbaues (fossile Bodenkomplexe, Umlagerungsvorgänge unterschiedlicher Intensität) war von vornherein klar, daß neben den bodenstratigraphisch-geomorphologischen Geländeuntersuchungen spezielle Untersuchungen des Substrats durchzuführen waren. Entsprechend der skizzierten Fragestellungen war dabei insbesondere das Substratgefüge - im weitesten Sinne - zur Klärung der wechselseitigen Beeinflussung geomorphologischer und pedologischer Prozesse sowie ihrer zeitlichen Relation bedeutungsvoll. Das dafür am besten geeignete Verfahren ist die mikromorphologische Dünnschliffanalyse.

Die in die mikromorphologische Analyse des Profils gesetzten Erwartungen haben sich voll erfüllt. Die Geländebefunde konnten in wesentlichen Punkten abgesichert werden; an einigen problematischen Stellen des Profils führte die Dünnschliffuntersuchung zu Ergebnissen, die eine genauere geomorphologische Interpretation zulassen. Es soll hier besonderer Wert auf die Feststellung gelegt werden, daß gerade das enge Zusammenspiel von Gelände- und Laboruntersuchung mit ständiger Ergebnisrückmeldung zu einem - gemessen am angestrebten Ziel — optimalen Erfolg führte. 


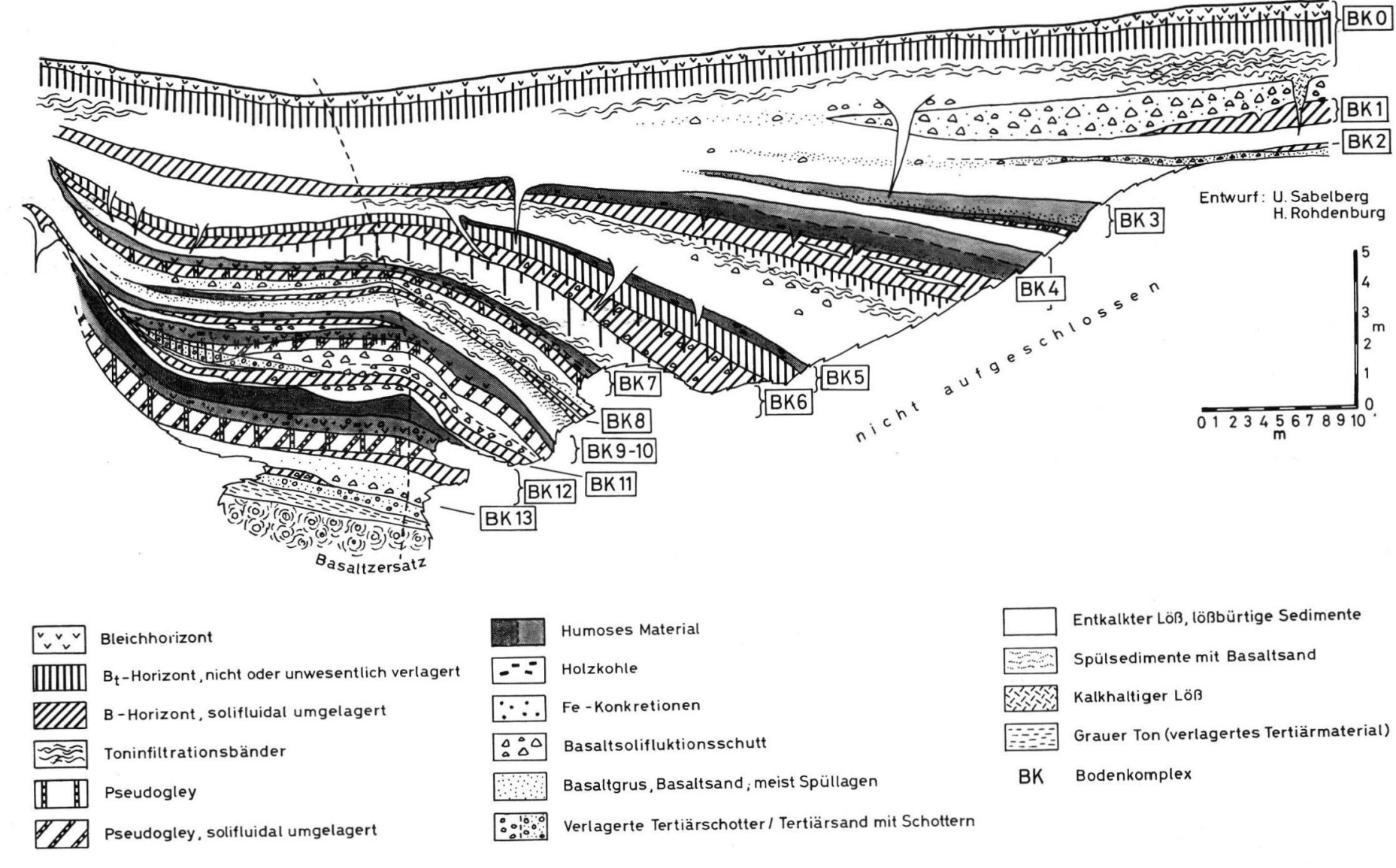

Abb. 2: Das Quartärprofil im Basaltbruch der Fa. Nickel, Dreihausen. 


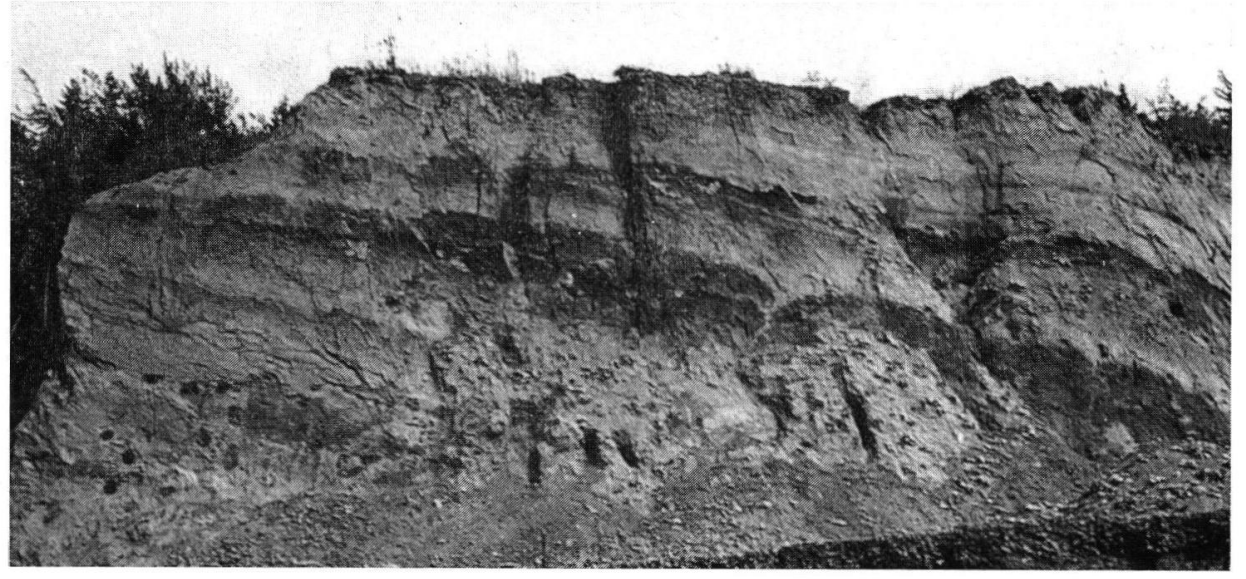

Abb. 3: Blick auf die in Abb. 1 sichtbare Profilwand mit den Bodenkomplexen BK 4 u. 5/6 und den schwächer entwickelten BK 7 und 8. Das Foto zeigt etwa die im mittleren'Drittel der Abb. 2 dargestellte Boden-Sediment-Folge. (Abb. 1 u. 3 nach Farbfotos von R. Huckriede, Marburg. Aufnahme: August 1973).

Wie schon früher betont (Rohdenburg \& Sabelberg 1973, Sabelberg \& Rohdenburg 1975), sind reich gegliederte Quartärprofile, die einen großen Teil des Quartärs umfassen, geomorphologisch stets als Seltenheit anzusehen. Überwiegende Akkumulation während längerer Zeit, d. h. über mehrere quartäre Klimazyklen, die g e n e r ell durch Tendenz zur Abtragung gekennzeichnet sind, ist nur in geomorphologischen Sonderpositionen möglich (Saelberg, Rohdenburg \& Havelberg 1974). Infolgedessen entfällt zunächst die Möglichkeit, unter diesen wenigen für die Quartärstratigraphie so bedeutsamen Profilen die hinsichtlich der Eindeutigkeit der zu gewinnenden Aussagen optimaleren auszuwählen. Als eine weitere Folge ergibt sich der weitgehende Mangel an stratigraphischen und auch faziellen Vergleichsmöglichkeiten beim derzeitigen Stand der Forschung. Es muß also nach Wegen gesucht werden, diese prinzipiellen Schwierigkeiten zu überwinden und durch geeignete Hypothesenbildung dennoch zu einem Konzept für die Quartärgliederung in Mitteleuropa insgesamt und für quartäre Warmzeit-Kaltzeit-Zyklen im einzelnen zu gelangen. Dies war ein wesentliches Motiv für die vorliegenden Untersuchungen.

Das Quartärprofil Dreihausen erwies sich für ein solches Vorhaben als sehr geeignet, da es aufgrund seiner geomorphologischen Situation im Abdachungsbereich des Vogelbergbasaltes gegen die Senke des Ebsdorfer Grundes (Rohdenburg 1968, SAbelberg, RohdenBURg \& Havelberg 1974) kein reines Lößprofil ist, ja nur in einzelnen Schichten überhaupt ausgeprägte Lößdominanz aufweist. Geomorphologische Prozesse der Kaltzeiten, Gelisolifluktion ebenso wie fluviale Spülprozesse, werden im Gegensatz zu reinen Lößprofilen durch die unterschiedlichen Beimengungen basaltischen Materials (Basaltsand, Basaltgrus, Basaltschutt) sowohl makroskopisch wie auch im Dünnschliff deutlich erkennbar und erlauben so eine sehr weitgehende prozessuale Differenzierung der Kaltzeiten.

Andererseits ergeben sich daraus auch neue Schwierigkeiten. Im Gegensatz zu reinen Lößprofilen (Semmel 1973, 1974; Kukla 1969), wo die interglazialen $B_{t}$-Horizonte an Standorten mit offenbar geringerer Abspülung und Gelisolifluktion überwiegend eindeutig autochthon sind, findet man im stark gelisolifluidal überprägten Profil Dreihausen von autochthonen fossilen Böden nur noch basale Reste. Meist sind die B-Horizonte verlagert; wenn auch ihre morphologischen Eigenschaften noch eine Ansprache erlauben, im mikro- 
skopischen Bild zeigen sie jedoch keine autochthonen Merkmale mehr. Durch die mikromorphologische Analyse war es aber möglich, die außerordentlich komplexe zeitliche Differenzierung von Bodenbildung und alternierender Geomorphodynamik in den interglazialen und frühglazialen Abschnitten der Zyklen, wie sie bereits durch Geländebeobachtungen festzustellen war (SAbelberg, Rohdenburg \& Havelberg 1974), detaillierter nachzuvollziehen (vgl. auch KuKLA 1969, Rohdenburg 1971: 299). Dadurch war es möglich, auch in Fällen, in denen kein e autochthonen Bodenreste mehr gefunden wurden, zu einem hohen Maß an Sicherheit in der stratigraphischen Ansprache und damit in der paläo-geoökologischen Bewertung einzelner Horizonte und Schichten des Profils zu gelangen.

Geomorphologische Analyse im Gelände und mikromorphologische Analyse im Labor zeigten unabhängig voneinander, daß in dem Profil - trotz seiner außerordentlich großen stratigraphischen Komplexität - nur sehr wenige Sedimenttypen und Bodenmerkmalstypen vertreten sind, die mit hoher Sicherheit genetisch interpretiert werden können; hierdurch ist eine enge Korrelation zwischen diesen Typen und bestimmten geomorphologischen oder pedologischen Prozessen bzw. ökologischen Grundzuständen gewährleistet. Die stratigraphischen Positionen der einzelnen Typen im Profil sind ganz offensichtlich nicht zufällig: sie wiederholen sich in regelhaft zyklischer Abfolge. Deshalb werden in den folgenden Abschnitten zunächst die mikromorphologisch unterschiedenen Merkmalstypen vorgestellt und dann in geomorphologisch-genetischer Interpretation zu einem Idealbild eines quartären Klimazyklus stratigraphisch vereinigt. Erst danach soll dann eine Beschreibung der individuellen Realisationsformen der einzelnen Zyklen im Profil folgen.

\section{Mikromorphologische Analyse}

Die mikromorphologische Analyse ermöglicht durch die Bestimmung von Gefügeund spezifischen pedogenetischen Merkmalen Aussagen über geomorphologische und Bodenentwicklungsprozesse. Zeitlich-stratigraphische Zusammenhänge kann sie demgegenüber primär nicht erfassen. Die im folgenden erläuterten mikromorphologischen Typen sind dementsprechend zunächst nur Gefügetypen. Erst nach entsprechender Interpretation sind sie mit bestimmten Prozeßtypen zu korrelieren. Nicht immer aber ist die Zuordnung von Gefügetyp und Prozeßtyp eindeutig und umkehrbar. Die gewählten Bezeichnungen dürfen deshalb auf keinen Fall als Ausdruck einer zeitlichen Abfolge verstanden werden. Die Synthese von Prozessen und ihrer zeitlichen Abfolge wird in den folgenden Abschnitten gegeben.

Die Ergebnisse der mikromorphologischen Untersuchungen des Quartärprofils Dreihausen zeigen im allgemeinen folgendes: Einzelne Abschnitte des Profils haben zum Teil ähnliche oder übereinstimmende mikromorphologische Merkmale; das gesamte Profil ist entkalkt, mit Ausnahme eines Eiskeils im jüngsten Abschnitt; auch die Schichten mit starker Lößdominanz enthalten fast alle eingewehtes oder eingespültes Bodenmaterial; fast in allen Abschnitten findet man Basaltgrus und seine Verwitterungsprodukte und außerdem Gefügemerkmale, die auf Umlagerungsvorgänge (Gelisolifluktion, Verspülung) hinweisen. Aufgrund der Ergebnisse lassen sich folgende mikromorphologische Typen unterscheiden (Abb. 4):

Typ a: Entkalkter, mehr oder weniger humoser Löß mit Holzkohle und Mangankonkretionen, charakterisiert durch eine hellbraune bis braune, meistens geflockte schluffreiche Grundmasse mit zahlreichen röhrenförmigen Hohlräumen (Nadelstichporen). Im Gefügeplasma sind häufig dunkle, umgelagerte verkohlte Pflanzenteile eingearbeitet. Zum Teil sind auch noch gut erhaltene rundliche zoogene Aggregate vorhanden. 
Der humose Löß enthält oft verschiedene Anteile von Fremdmaterial, so daß Typ a in 4 Subtypen unterteilt werden kann, und zwar nach Art und/oder Menge des Fremdmaterials.

$\mathrm{Sub}$ t y p a 1 : Humoser Löß mit geringem Basaltanteil, dagegen reich an Holzkohle und z. T. mit noch gut erhaltenen zoogenen Aggregaten (Tafel I, Fig. 1).

$\mathrm{Subtyp}$ a 2 : Ähnlich wie a1, aber mit starker Beteiligung der basaltischen Komponente (Tafel I, Fig. 2).

$\mathrm{Sub}$ t y $\mathrm{p}$ a 3 : Im allgemeinen ist das Material einem schwach humosen Löß ähnlich, dabei treten aber Merkmale einer starken Umlagerung in den Vordergrund. Gefügeteile aus einem $\mathrm{B}_{\mathrm{t}}$-Horizont (Feintonanlagerungen) sind mit humosen Partien oder mit umgelagerten Resten eines Braunlehms vermengt (Tafel I, Fig. 3).

Subtyp a 4 : Die Sandkomponente überwiegt; Korngrößen und petrographische Zusammensetzung sind sehr unterschiedlich (Tafel I, Fig. 4).
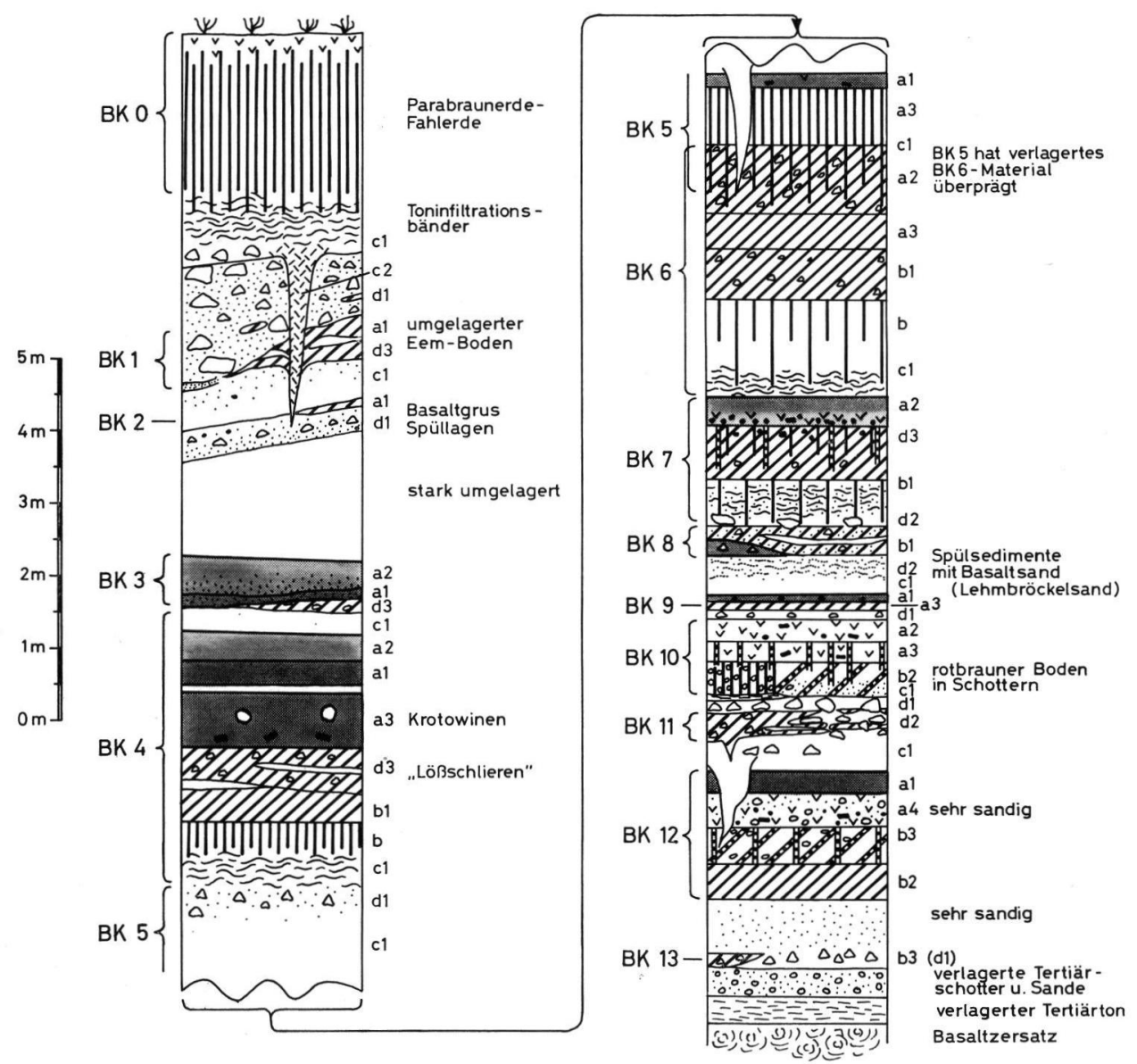

Abb. 4: Stratigraphische Abfolge und mikromorphologische Typen. (Die an der rechten Seite der Profile stehenden Symbole kennzeichnen die mikromorphologischen Typen bzw. Subtypen. Signaturen vgl. Abb. 2.) 
Typ b: Lößmaterial mit stark ausgeprägten $B_{t}-M e r k m a l e n$. In der schluffreichen lößbürtigen Grundmasse befinden sich zahlreiche eingemischte Reste von Braunlehmteilplasma oder von braunlehmartigen Böden; stellenweise sind sie vermengt mit hellgrauen Schluffzonen (wahrscheinlich umgelagertes $\mathrm{A}_{\mathrm{l}}$-Material) und mit Basaltstückchen oder Sandkörnern (Tafel II, Fig. 1). Die Merkmale einer gelisolifluidalen oder fluvialen Verlagerung sind deutlich erkennbar. Typ b wurde in drei Subtypen unterteilt, und zwar:

Subty p b 1 : Lößmaterial mit eingemengten Resten eines $\mathrm{B}_{\mathrm{t}}$-Horizonts. Diese sind deutlich gelisolifluidal verlagert. Darin finden sich nebeneinander dicke Tontrümmer von Belägen oder von einem ehemaligen braunlehmartigen Boden, sowie schluffreiche, tonverarmte Partien und Basaltgrus (Tafel II, Fig. 2).

$\mathrm{Sub}$ y p b 2 : Zum Teil feinkörniger Löß, vermischt mit umgelagertem $\mathrm{B}_{\mathrm{t}}$-Material; das Ganze ist nachträglich durch Pseudovergleyung überprägt worden (Eisen- und EisenMangan-Ausscheidungen in Streifen oder Flecken und saumartig in Hohlräumen (Tafel II, Fig. 3).

$\mathrm{Subty} \mathrm{p} \mathrm{b3}$ : Auch hier sind umgelagerte $\mathrm{B}_{\mathrm{t}}$-Reste sehr stark vertreten, es überwiegen aber hohe Sandanteile und Anzeichen fluvialer Prozesse (Tafel II, Fig. 4).

Ty p c: Lößleh $\mathrm{m}$ im Sinne eines primär überwiegend äolisch gebildeten Materials, das sekundär durch eluviale und pedogene Prozesse überprägt wurde.

Subty p c 1 : Schluffreiches Material mit pedogener Überprägung. Nadelstichporen charakterisieren das Hohlraumsystem; ehemalige Tiergänge sind wieder verfüllt. Tonige Substanzen umhüllen die größeren Schluffkörner oder sind in Hohlräumen akkumuliert. Gerundete dunkle Fe-Mn-Konkretionen sind im Plasma eingemengt; der Basaltanteil ist sehr unterschiedlich. Auch trümmerartige Tonbeläge sind im Innengefüge eingearbeitet, während Tonbeläge in situ selten vorkommen.

S u b t y p c 2 : Im allgemeinen dem Subtyp c1 ähnlich, aber carbonathaltig. Neben primären Carbonaten ist der mikrolithische Calcit im ganzen Gefügeplasma zu finden; er tritt auch krustenartig in den Nadelstichporen auf. Die Calcitkrusten sind fast alle mit Feinton überzogen; in den Calcitkrusten und im Feinton sind Eisen- und Manganoxide ausgefällt.

Typ d: Basaltzersatz, \pm stark verwittertes basaltisches Material, z. T. ist Fremdmaterial eingemischt.

Subtyp d 1: Grusig-sandiger Basaltzersatz, bestehend aus kleinen, fast kugelförmigen Basaltstückchen mit vielen Rissen und dünnen wandständigen Tonbelägen (Tafel III, Fig. 1). Der Basaltgrus ist sehr oft in eine hellbraungelb gefärbte tonige Substanz eingebettet (Tafel III, Fig. 2).

S ubty p d 2 : Basaltzersatz mit meist humosem Löß; der Basaltgrus bzw. Basaltsand in verschiedenen Phasen der Verwitterung (Anteil 70 bis $80 \%$ ) ist mit kleinen Lößbröckeln vermischt (Tafel III, Fig. 3).

Subty p d 3 : Basaltzersatz in toniger Grundmasse; bei schwacher Vergrößerung erscheint diese Schicht als eine ziemlich gleichmäßige Tonmasse (Tafel III, Fig. 4), in der stark verwitterte Basaltstückchen eingeschlossen sind. Bei stärkerer Vergrößerung ist aber zu erkennen, daß das tonige Plasma aus gelblichen, stark doppelbrechenden Feintonaggregaten besteht, die wahrscheinlich durch Umlagerung gerundet wurden. 


\section{Der Idealzyklus}

Zum Wesen eines Ideals gehört es, daß reale Erscheinungsformen ihm stets nur zum Teil entsprechen. Bei den 13 im Profil Dreihausen ausgeschiedenen Zyklen gleicht kein Zyklus dem anderen. Dennoch scheinen alle nur nach e in e m Grundschema aufgebaut zu sein, was allerdings für einige Zyklen nicht unmittelbar, sondern erst bei synoptischer Betrachtung deutlich wird. Das augenfälligste Gliederungskriterium, basaler Bodenkomplex-bodenfreie Sedimente im Hangenden, ist bei sämtlichen Zyklen gut faßbar. Alle anderen Kriterien treffen jeweils nur für eine Gruppe, einige sogar nur für einen Zyklus zu. Der Entwurf eines Idealzyklus erfordert also die gedanklich-hypothetische Uberbrückung von Lücken und das mosaikartige Zusammenfügen von Einzelbefunden. Das wirft die Frage nach der Rechtfertigung für ein solches Vorgehen auf: Was gibt Anlaß zu der Annahme, die individuelle Erscheinungsform der einzelnen Zyklen sei $\mathrm{n}$ i c h t Ausdruck eines jeweils individuellen Entwicklungsganges, sondern nur Ausdruck jeweils lokal z ufälliger „Überlieferungs“-Bedingungen? Oder positiv gefragt: Was ist Anlaß zur Bildung der Hypothese, allen Zyklen liege der gleiche Entwicklungsgang oder die gleiche Abfolge pedologischer und geomorphologischer Prozesse zugrunde? Zunächst: Das Auftreten von zum Teil beträchtlichen Überlieferungs-(Schicht)-Lücken ist unbestreitbar. Dies manifestiert sich deutlich zum Beispiel dort, wo ein reich gegliederter Komplex seitlich in einen nur noch geringmächtigen, kaum gegliederten übergeht und einem Komplex vergleichbar wird, der überhaupt nur in der "rudimentären“ Form im Profilanschnitt zu sehen ist, (BK 4 - BK 1, vgl. auch Angaben zu BK 1 in Sabelberg, Rohdenburg \& Havelberg 1974). In den seltenen Fällen aber, in denen in e in e m Profil (oder in mehreren Profilen mit eindeutigem stratigraphischen Bezug) mehrere verschieden alte Zyklen sehr vollständig erhalten sind, zeigt sich stets das gleiche Phänomen: je vollständiger die Zyklen erhalten sind, desto ähnlicher sind sie in ihrem $\mathrm{A}$ u f b a u. Seit längerem bekannt sind die Beispiele aus den berühmten Lößprofilen der Tschechoslowakei (KuKLA 1969), wo mindestens für die letzten 3 Zyklen weitestgehende Gleichartigkeit des Aufbaues nachgewiesen ist, für die älteren Zyklen mindestens prinzipielle Übereinstimmung. Aus eigenen Untersuchungen kennen wir das Beispiel des Lößprofiles bei Ostheim (SABELBERG et al. 1974), wo ebenfalls die 3 jüngeren Zyklen so weitgehende Äquivalenz des Aufbaues zeigen, daß der Schluß auf eine generell gleichartige geoökologisch-geomorphologische Entwicklung in allen 3 Zyklen geradezu zwingend wird (Abb. 5). Die Untersuchungen von BiBus (1974) zur Gliederung rißzeitlicher Lößserien in Südhessen bestätigen eindrucksvoll die bis ins einzelne gehende Parallelität zur bekann-

Tafel I

Fig. 1: Subtyp a1: Schwach humoser Löß mit umgelagerter Holzkohle (Mitte) und dunkle, fast schwarze Mangan-Konkretionen (rechts und links oben); $32: 1$.

Fig. 2: Subtyp a2: Übersichtsbild; humoser Löß mit starker Basaltbeimengung (weiß Basaltsand); $5: 1$.

Fig. 3: Subtyp a3: Schwach humoser Löß mit dünnen Tonbelägen in den Nadelstichporen, Tontrümmer (rechts oben) und fleckige Mangan-Ausscheidungen; 32:1.

Fig. 4: Subtyp a4: Ubersichtsbild; umgelagertes, humoses schluffreiches Material, mit Sand vermischt; $5: 1$. 


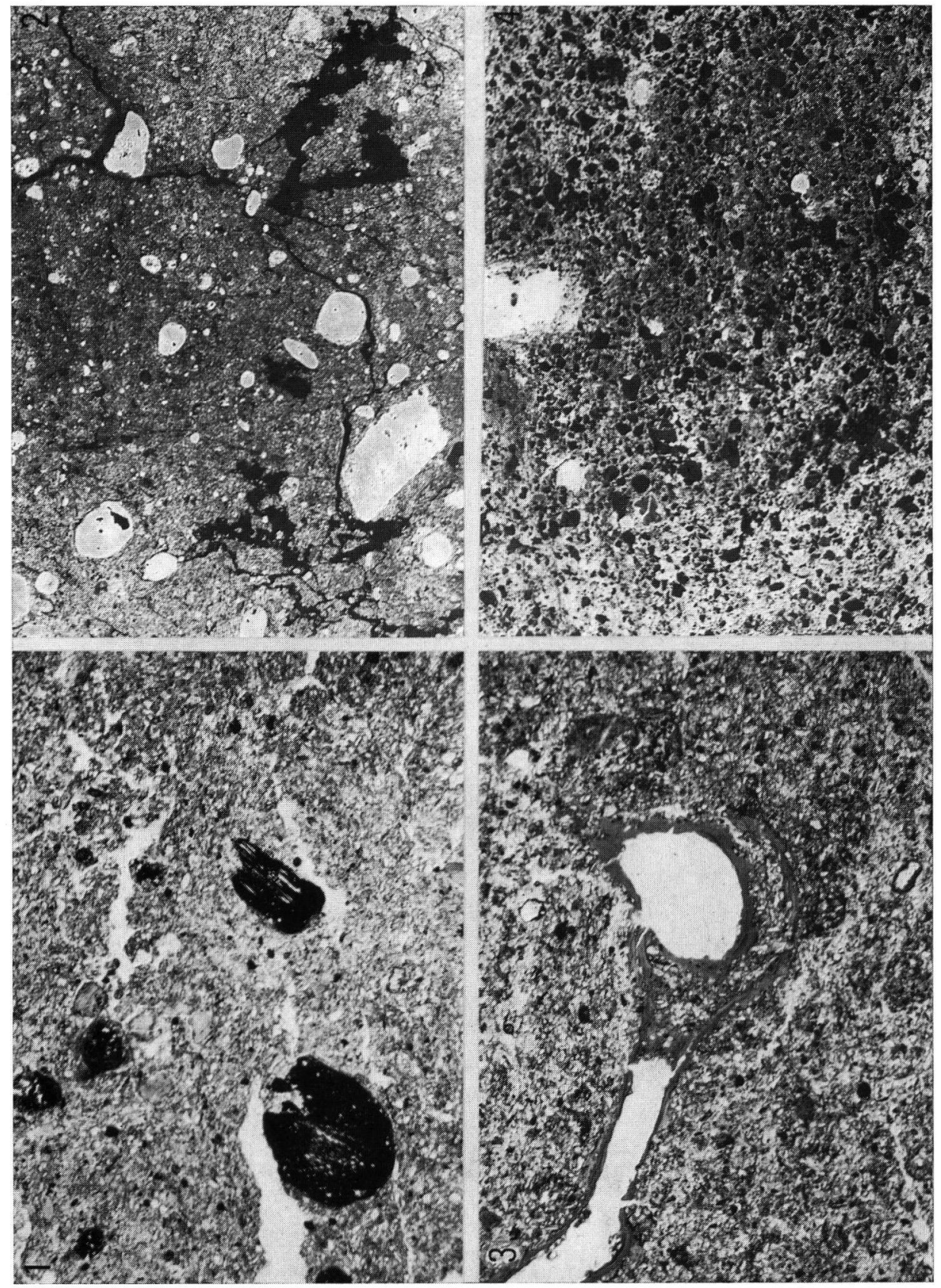




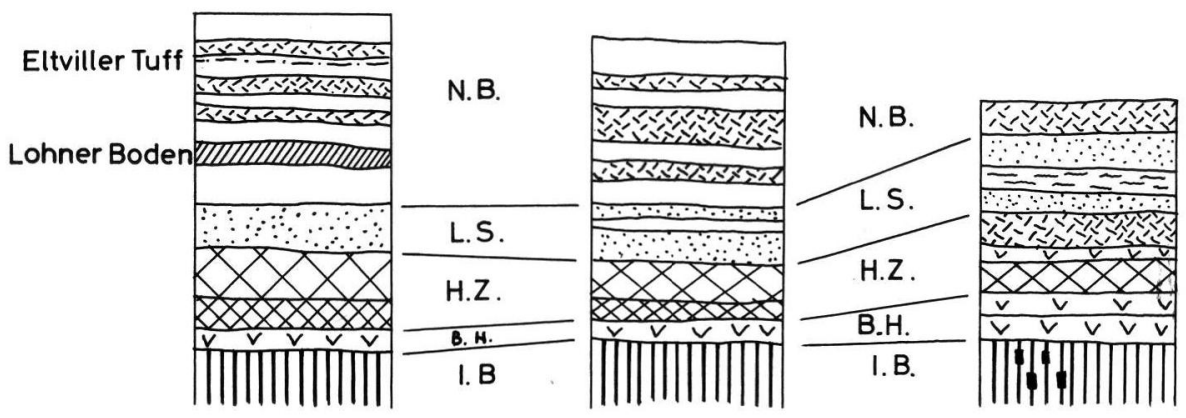

\section{|ाل Parabraunerde, teils pseudovergleyt}

\section{$v_{v}^{v}$ Bleichhorizont}
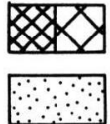

Humusböden, humose Sedimente

Lehmbröckelsand
Brauner Boden

Naßböden

Helle Schwemm sedimente

Löß

I.B. Interglazialer Boden; B.H. Frühglaziale Bleichhorizonte; H.Z. Frühglaziale Humuszonen; L.S. Zone der frühglazialen Schwemmsedimente und Lehmbröckelsande; N.B. Zone der mittel- und jungglazialen Naßböden in Löß

Abb. 5: Gleichartigkeit des Aufbaues der drei jüngeren Zyklen von Ostheim. Entwurf: U. SABELBERG.

ten Gliederung der Würmlösse. Von diesen Erfahrungen ausgehend, kann für alle quartären Klimazyklen - zumindest aber für die jüngeren - ein gleichartiges Grundschema der Entwicklung angenommen werden. Die Überprüfung, ob ein solches Schema auch für die älteren Zyklen in allen Fällen ausreichende Erklärung der auftretenden Phänomene gewährleistet, ist nachher leicht durchführbar, wie am Beispiel des Profils Dreihausen gezeigt werden kann.

\section{Tafel II}

Fig. 1: Subtyp b: Umgelagertes Parabraunerde-Material; deutlich geschichtete Tonbeläge, schluffreiche Partien mit blankgewaschenen Mineralkörnern (keilförmige helle Schmitze oben) und Mangan-Hydroxid-Krusten (dunkelste Partien); $32: 1$.

Fig. 2: Subtyp b1: Übersichtsbild; solifluidal umgelagertes Material; schluffreiche, tonarme Partien (dunklere Streifen), tontrümmerreiche Lagen (hellere Streifen) und tonreiche Grundmasse mit Basaltstückchen (rechts und links unten); $5: 1$.

Fig. 3: Subtyp b2: Ubersichtsbild; toniges braunlehmartiges Material mit Pseudovergleyung, diffus begrenzte Eisen-Mangan-Ausscheidungen (dunklere Flecken); rechts oben ein stark doppelbrechender wandständiger Tonbelag; $5: 1$.

Fig. 4: Subtyp b3: Übersichtsbild; in Sand (schwarz) eingeschlämmtes braunlehmartiges Material; $5: 1$. 


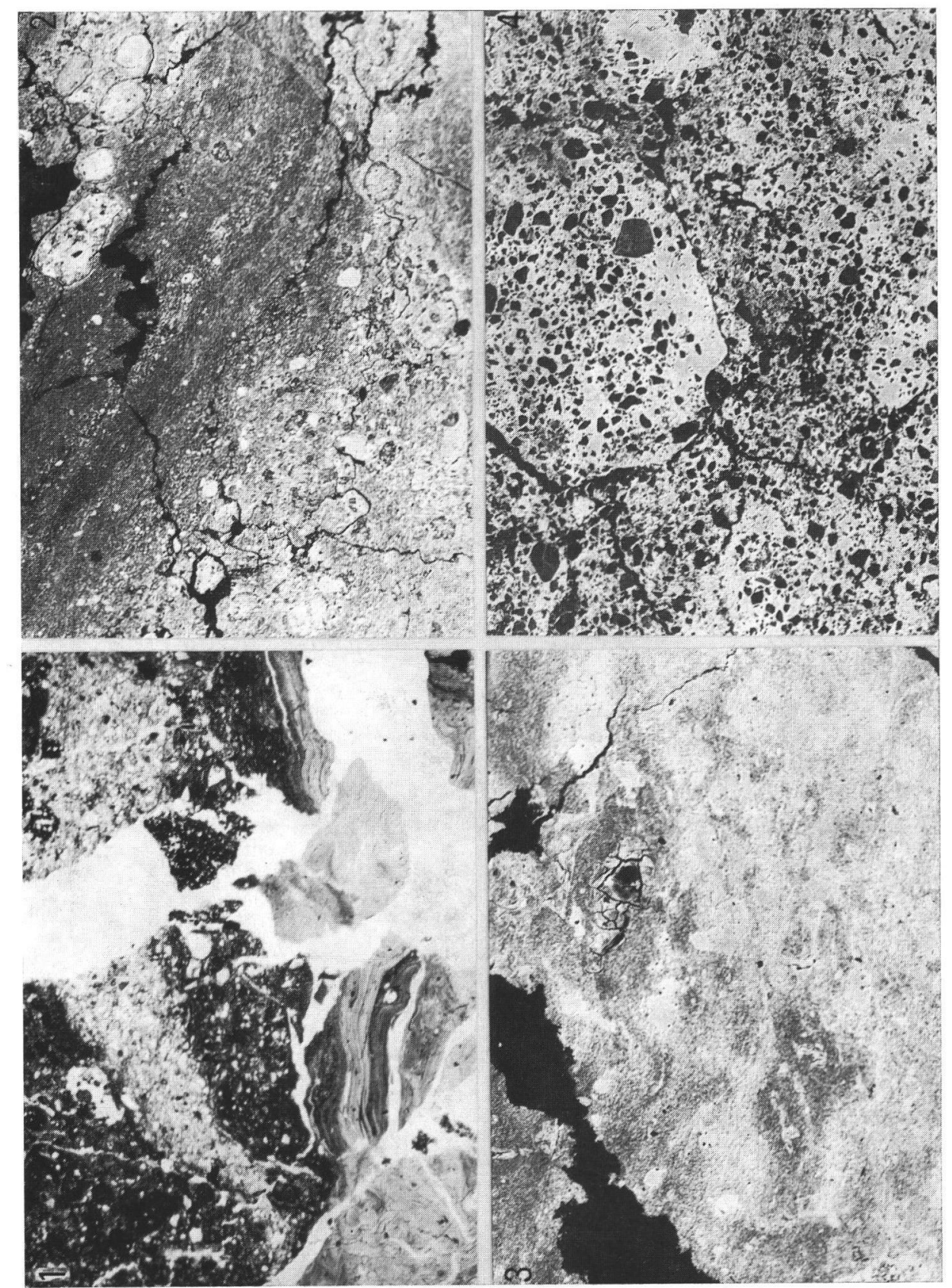


Aufgrund der Befunde des Profils Dreihausen wurde die Idealfolge eines quartären Klimazyklus (Abb.6) entworfen. Der Zyklus beginnt mit einem Interglazial, während dem sich aus allen geeigneten Substraten (d. h. vorzugsweise aus Löß und lößartigen Sedimenten) eine stark ausgeprägte Parabraunerde mit charakteristischem Toninfiltrationsbänderhorizont im Liegenden bildet. In basaltischem Substrat (Basaltgelisolifluktionsdekken) treten die Merkmale der Tonverlagerung zurück, wahrscheinlich wegen des sehr hohen Tongehalts des Basaltverwitterungslehms (Phase I, Subtypen b1 u. d3 ohne Umlagerung). Das Ende des Interglazials ist angezeigt durch eine erste, noch wenig wirksame Phase des Bodenfließens. Es werden meist nur die oberen Dezimeter der Bodendecke laminar verschleppt. Die Bewegungsweiten können über wenige Meter kaum hinausgegangen sein, da makroskopische Gefügemerkmale der Böden häufig noch gut erkennbar sind. Im Dünnschliff macht sich dies allerdings schon als nachhaltige Zerstörung des Feingefüges bemerkbar. Häufig von der vorhergehenden Parabraunerdebildung kaum zu trennen (und wohl in solchen Fällen auch als Parabraunerde-Weiterentwicklung aufzufassen), manchmal aber auch durch das beschriebene Gelisolifluktionsereignis deutlich getrennt, macht sich nachfolgend eine Phase kräftiger Pseudovergleyung bemerkbar. Ein neuer Gelisolifluktionsschub überprägt auch diese Bodenbildung (Phase IIa). Anschließend kommt es wieder zu einer sehr kräftigen Pseudovergleyung (Phase IIb, Subtyp b2). Ob der im Hangenden meist ausgebildete kräftige Bleichhorizont genetisch mit dem Pseudogley zusammenhängt, kann zunächst nur vermutet werden; denn das Substrat des Bleichhorizonts erweist sich als neues Sediment, und zwar aufgrund eines häufig sehr hohen Anteils an Basaltgrus, der als fluviales Sediment geringer Transportweite anzusehen ist. Charakteristisch sind Holzkohlestückchen und die Anreicherung von Eisen-Mangan-Konkretionen. Vor allem der letzte Befund legt nahe, dieses Sediment als fluvial umgelagerten oberen Teil des liegenden Pseudogleys zu deuten (Phase IIc, Subtyp a2). Andererseits ist in einigen Fällen auch dieser Pseudogley bereits (teilweise) in einem neuen Schwemmsediment entwickelt (Abb. 6, Variante B links). Einzelne kleine eiskeilähnliche Formen im Bleichhorizont (ebenso wie die basale, schwache Gelisolifluktion) belegen bereits zu dieser frühen Zeit das kurzfristige Auftreten kaltzeitlicher Umweltbedingungen. Noch deutlicher wird dies durch das Auftreten von Gelisolifluktionsdecken größerer Wanderweite (Abb. 6, Variante B); durch die Gelisolifluktion wurde zum Teil - wenn auch nur lokal begrenzt - bereits die gesamte interglaziale Bodendecke abgeräumt, in Kuppenlagen bis auf den anstehenden Basalt, wie die Basaltschuttbeimengung beweist (Phase II d1, Variante B, Subtyp d2). In der folgenden Phase kommt es zur Ablagerung fluvialer Sedimente (mit Holzkohle), deren teilweise

Tafel III

Fig. 1: Subtyp d1: Ubersichtsbild; grusig-sandiger Basaltzersatz in verschiedenen Phasen der Zersetzung, z. T. in fast farblosen Ton eingehüllt (grau); $5: 1$.

Fig. 2: Subtyp d1: Vergrößerter Ausschnitt aus Fig. 1 - deutlich die hellen ungeschichteten Tonpartien zwischen verwitterten Basaltbröckchen (schwarz); im Bild links ein geschichteter, kräftig gefärbter pedogener Tonbelag; $32: 1$.

Fig. 3: Subtyp d2: Ubersichtsbild, Basaltgrus (hell) mit humosen Lößbröckeln (dunkler); 5 : 1 .

Fig. 4: Subtyp d3: Ubersichtsbild; stark verwitterte, rundliche Basaltstücke (grau), in eine tonige Masse eingeschlossen (Basaltverwitterungslehm ?); $5: 1$. 

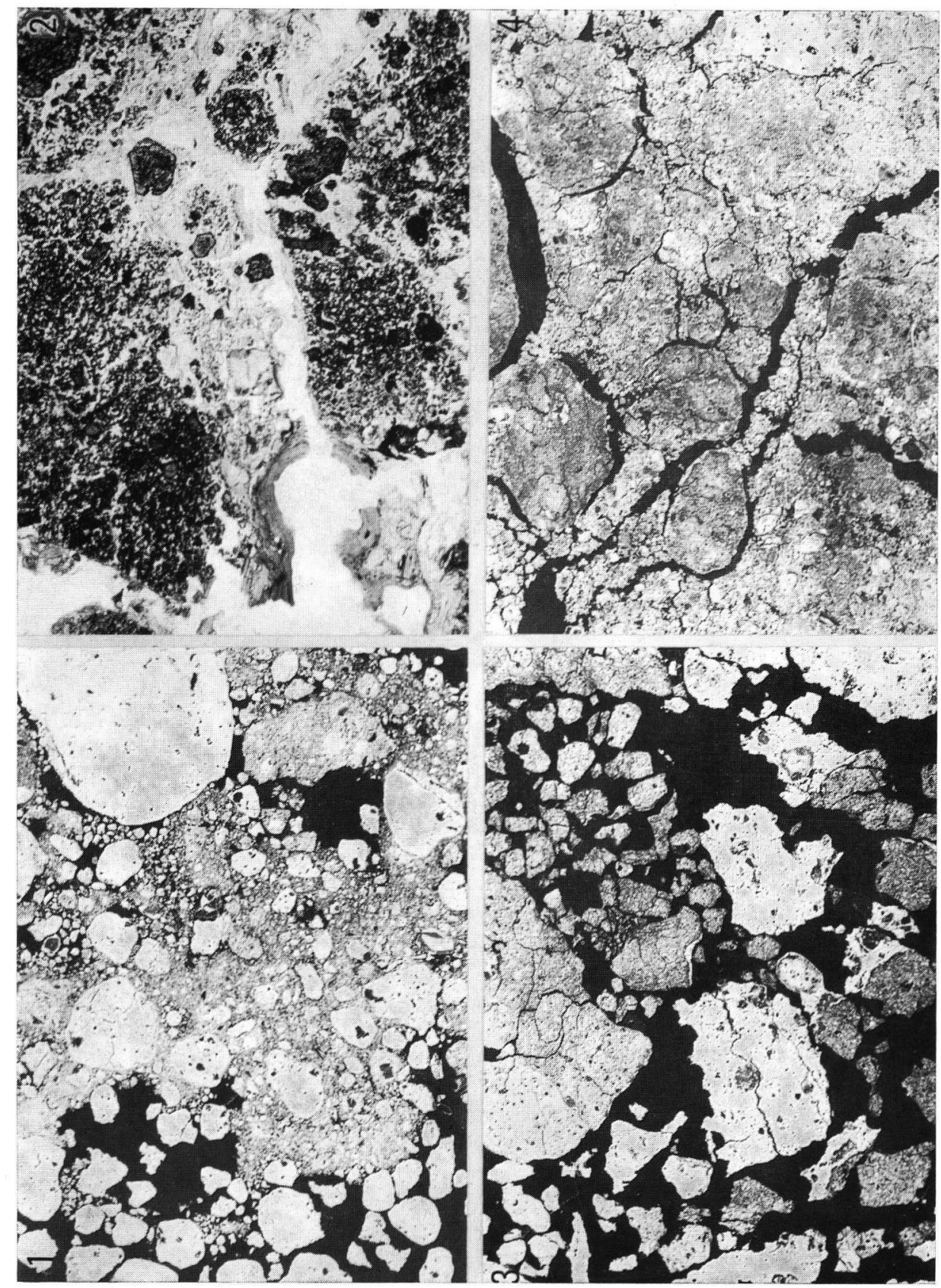


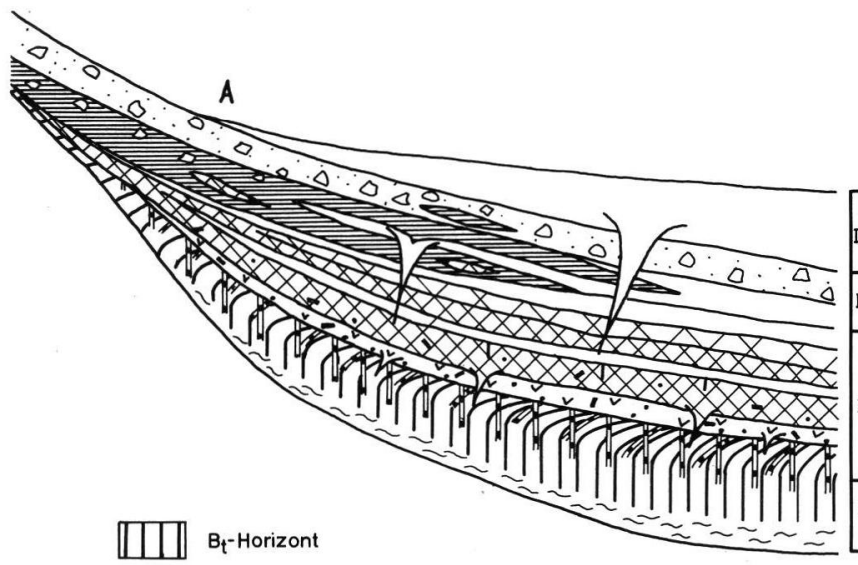

D.

鴋 Toninfiltrationsbänder

¿va Bleichhorizont in Spülsedimenten

D) B $_{\mathrm{t}}$-Horizont parautochthon (Gelisolifluktion)

Pseudogley parautochthon (Gelisolifluktion)

X Ah-Horizont (par-) autochthon

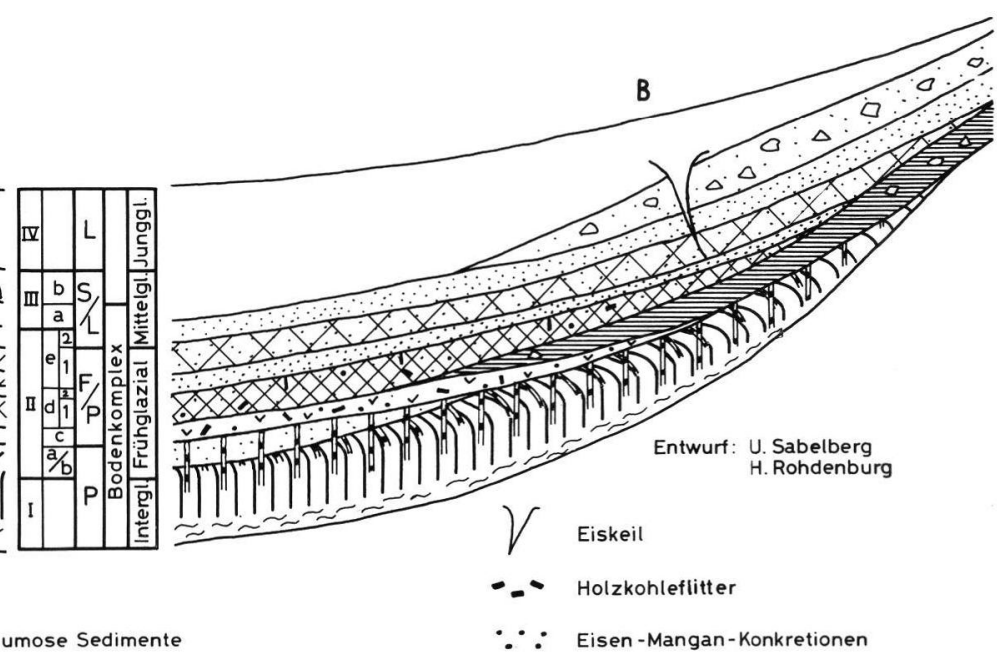

$\because \because$ Eisen-Mangan-Konkretionen

L Überwiegend äolische Dynamik

S Überwiegend Gelisolifluktion

F Überwiegend fluviale Dynamik

$\mathrm{P}$ Überwiegend Pedogenese

Abb. 6: Idealfolge eines quartären Warmzeit-Kaltzeit-Zyklus (nach Befunden im Profil Dreihausen).

I. Interglaziale Parabraunerde in Löß oder Basaltsolifluktionsdecken.

II. a) Schwache Gelisolifluktion, später Tonverlagerung und Pseudovergleyung;

b) Erneut gelisolifluidale Verlagerung und Pseudovergleyung;

c) Verspülung und Akkumulation, Bleichhorizontbildung, Gelisolifluktionsdecke aus I, siehe aber auch Variante A;

d) Schwemmsedimente, teilweise schon Löß, darin Humusanreicherung und schwache Tonverlagerung;

e) Stärkere Umlagerung (gelisolifluidal, fluvial) mehr Löß, humos, häufig Basaltgrus.

III. a) Gelisolifluktionsdecke aus I+II, siehe aber Variante B;

b) Gelisolifluktionsdecke aus Basaltgrus, Löß und frischem Basalt.

IV. Überwiegend Lößsedimente, teilweise mit höherem Basaltgrusanteil. 
hoher Schluffgehalt auf Anwehung von Lößstaub schließen läßt. In diesem neuen Sediment entwickelt sich ein sehr kräftiger Humusboden, in dem stellenweise ein kurzzeitiges Aufleben der Tonverlagerungsdynamik erkennbar wird (Phase II d1, Subtypen a1, a3 u. a4). Ob die kleinen Eiskeile, die aus dem Sedimentkörper in das Liegende hineinreichen vor oder nach der Bodenbildung entstanden sind, kann nicht eindeutig gesagt werden. Eine nachträgliche Bildung dürfte aber die größere Wahrscheinlichkeit besitzen. Nach der Bodenbildung II d1 kommt es erstmals verbreitet $\mathrm{zu}$ fast reiner Lößablagerung, allerdings überwiegend wohl in Form von Schwemmlöß, denn in Bereichen mit anstehendem Basalt im Einzugsgebiet ist der Basaltgrus-Anteil beträchtlich (Phase II d2). In der darüber folgenden Humuszone, die nochmals gegliedert sein kann, ist der Humusgehalt meist deutlich geringer, er nimmt nach oben ab. Tonverlagerung ist nicht mehr feststellbar. Hinweise auf Umlagerung gewinnen an Bedeutung (Phase II e1). Eine nicht sehr mächtige Lößakkumulation (Phase II e2) schließt die Serie ab.

Zusammenfassend betrachtet ist die Hauptphase II sehr deutlich durch mehrere Bodenbildungen gekennzeichnet, zunächst in feuchterer Variante (Pseudogleye), dann mit zunehmend trockeneren Formen (Schwarzerdeartige Böden, schwächere Humusböden). Gelisolifluktion spielt als Umlagerungsprozeß, außer während kurzer Phasen am Anfang, nur eine untergeordnete Rolle; die dazu notwendigen Temperaturerniedrigungen wurden wohl zunächst nur kurzzeitig erreicht. Dafür spricht auch, daß bis in die HumuszonenZeit noch Gehölzpflanzen verbreitet gewesen sein müssen (Holzkohle!). Wo Gelisolifluktion wirksam wurde, trat sie, insbesondere während der Humuszonen-Zeit, in Form sehr langsamen laminaren „Fließens“ auf, das so gleichmäßig verlief, daß ganze Bodenhorizont- und Sedimentabfolgen unter Beibehaltung ihrer gegenseitigen Orientierung gleichmäßig ausgedünnt wurden (Phase IId, Variante A ganz links).

Der vorherrschende Umlagerungsprozeß ist im übrigen die Verspülung, sie erweist sich aber insgesamt auch nur als bedingt leistungsfähig. Verspült wird vergruster Basalt, wo er zur Verfügung steht (Variante B), kaum jedoch B-Horizont-Material, wohl wegen der zu hohen Kohäsion. Überwiegend wird Löß von den Spülprozessen erfaßt, der zur Zeit der Humuszonen, aber zum Teil auch schon früher (vor der Gelisolifluktionsdecke II d1, Variante $B$ ) bereits angeweht wurde.

In einleuchtender Analogie zu den Verhältnissen in der Würmkaltzeit bezeichnen wir die geschilderte Hauptphase II als „Frühglazial“. Mit der folgenden Hauptphase III treten keine erkennbaren Bodenbildungen mehr auf. Dieser Abschnitt der Zyklen ist eindeutig durch Gelisolifluktionsdecken mit wechselnden Mengen an eingewehtem Material gekennzeichnet. Die ersten Gelisolifluktionsereignisse räumen - wo noch vorhanden mit den Resten interglazialer Bodendecken radikal auf. Die resultierende Gelisolifluktionsdecke (Phase IIIa, Variante A, Subtyp b1) enthält eine Mischung von B-Horizontresten, Humusbodenfetzen und Basaltschutt. Häufig tritt sie auch in geteilter Form mit wenig mächtiger Lößeinschaltung auf.

Die stratigraphische Position dieser Decke war zunächst hypothetisch rekonstruiert worden, basierend auf der Analyse ihrer makroskopisch und mikromorphologisch faßbaren Sedimentanteile. Nirgends im Profil war sie etwa auf einer Humuszone angetroffen worden, sondern stets nur (etwa entsprechend Variante A, ganz links) ohne interpretierbare Basisfolge. Erst später fand sich außerhalb des Hauptprofils eine Stelle, wo der Anschnitt eines eemzeitlichen, verfüllten Tälchens eine zweifelsfreie Ansprache erlaubte: In Hangposition fand sich in gewohnter Weise Solifluktionsmaterial aus dem Eem- $\mathrm{B}_{\mathrm{t}}-\mathrm{Hori}_{-}$ zont unter Löß und gelisolifluidal verlagerter Humuszone (vgl. Phase II). In Muldenposition des gleichen Tälchens überlagerte eine Gelisolifluktionsdecke (entsprechend Phase IIIa, Variante A Mitte) aus Eem-Bodenmaterial die dort nur wenig verlagerte Humuszone. 
Die Decken oberhalb IIIa (Phase IIIb) sind frei von Bodenmaterial, da dieses inzwischen völlig ausgeräumt war. In Bereichen, in denen die Böden bereits früher beseitigt wurden (Variante B), setzt entsprechend auch die Gelisolifluktion sogleich mit Basaltschutt ein. Eine Zuordnung zu Phase IIIa oder IIIb ist daher nicht immer eindeutig möglich. Wechselnd, von fast fehlend bis weitaus dominierend, ist der Lößanteil, besonders in der oberen Basaltgelisolifluktionsdecke.

Die skizzierte Hauptphase III mit Vorherrschen der Gelisolifluktionsdecken bezeichnen wir als „Mittelglazial“. Bemerkenswert ist, daß bei der von uns zunächst allein vorgenommenen Ausgliederung von „Bodenkomplexen“ selbst Teile dieser Phase III in Form von Gelisolifluktionsdecken mit hohem Bodenmaterialanteil noch miterfaßt, ja, in den mehrfach auftretenden Fällen stärkerer Profilreduktion allein erfaßt wurden und dann unter den vorläufigen Arbeitsbegriff „Interglazial-Äquivalente" fielen. Es ist jetzt sicher, daß diese Ansprache nicht zutreffend war und Mißverständnisse hervorrufen mußte. Andererseits ist hervorzuheben, daß das Auftreten solcher Decken die Existenz einer unmittelbar vorausgehenden Interglazialzeit sehr wohl anzeigt. Die Annahme, das Material verschiedener solcher Decken könne trotz deren unterschiedlicher stratigraphischen Position von ein und demselben Boden abstammen, würde es notwendig machen, die Erhaltung solcher Böden auch in steileren Relieflagen zu fordern, obwohl zwischenzeitlich Phasen mit voller geomorphodynamischer Aktivierung im gesamten Relief mit Sicherheit eingetreten waren. Das erscheint aber wenig einleuchtend.

Der von uns als "Jungglazial“ bezeichnete Abschnitt IV ist generell durch fast reine Lößakkumulation (Typ c1, c2) ausgezeichnet. Nicht überall - entsprechend den Reliefund Expositionsverhältnissen zur Ablagerungszeit - ist diese meist homogen wirkende Lößdecke in größerer Mächtigkeit entwickelt, verschiedentlich kann sie auch völlig fehlen. ob wegen nachfolgender Abtragung oder aus Mangel an Akkumulation, ist nicht zu entscheiden. Im Bereich dieser Lößdecke setzen die meisten und die größten Eiskeile an. Aufgrund dieser Beobachtungen ist wohl die Deutung zulässig, den jeweiligen Jungglazialabschnitt generell als die kälteste und trockenste Phase der Zyklen anzusehen. Die jungglazialen Lößdecken - zumal wenn sie nicht mächtig entwickelt sind - wurden häufig vollständig von der nachfolgenden interglazialen Bodenbildung überprägt. Dadurch wurden sie zunächst leicht übersehen. Gerade hier aber erwies sich wieder der Wert der Dünnschliffanalyse, die auch bei starker pedogener Überprägung eine Sedimentansprache ermöglicht.

Der hier vorgestellte Idealzyklus ist zunächst aus den Befunden des Profils Dreihausen in der Weise abgeleitet worden, daß er alle auftretenden Einzelbefunde in ein widerspruchsfreies, kausal befriedigendes „Minimalsystem“ einordnet, d. h. daß die Zahl der Einzelereignisse innerhalb des Systems so lange nicht erhöht wird, bis dies durch neue Beobachtungen erzwungen wird. Der Wert eines solchen Systems als brauchbare Hypothese erweist sich im vorliegenden Fall allerdings erst dadurch, daß es eine kausale Interpretation a $11 \mathrm{e} \mathrm{r}$ auftretenden realen Zyklen erlaubt - also insbesondere auch der Zyklen mit stark reduzierter Abfolge. Diese „Rückanwendung“ des gewonnenen Idealzyklus soll im folgenden Abschnitt erläutert werden.

\section{Interglazial-Glazial-Zyklen}

Die sich aus dem Vergleich mit der Idealfolge ergebenden stratigraphischen Bezüge der im Profil Dreihausen ausgegliederten Klimazyklen sind in Abb. 7 zusammengefaßt. Diese bildet mit den Angaben zum mikromorphologischen Typ in Abb. 4 die Grundlage für die folgende Übersicht. 

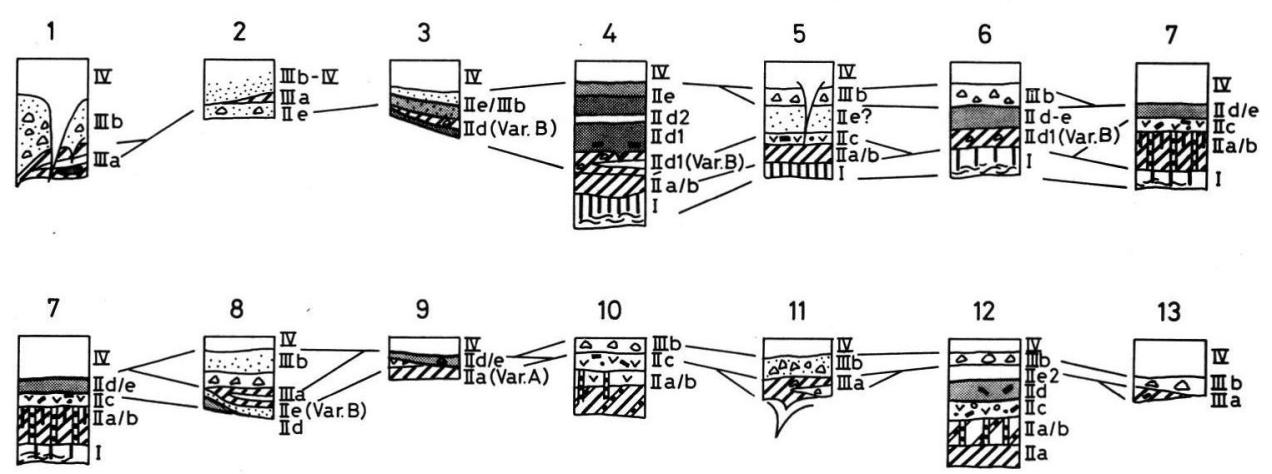

Abb. 7: Stratigraphischer Vergleich der Interglazial-Glazial-Zyklen im Profil Dreihausen (Signaturen vgl. Abb. 2). Entwurf: U. SABELberg.

Der holozäne Boden

An der Oberfläche der gesamten Serie ist eine kräftige holozäne Parabraunerde-Fahlerde von ein bis eineinhalb Meter Mächtigkeit entwickelt, die nicht oder nur geringfügig anthropogen gekappt ist.

Die holozäne Parabraunerde-Fahlerde (BK O) ist, da sie als einzige vollständig autochthon ist, ein wichtiger Vergleichsstandort für die Bewertung der älteren, umgelagerten Bodenreste. Allerdings trifft das insofern nur mit Einschränkung zu, als die Parabraunerde-Fahlerde als einziger Boden des gesamten Profils vollständig in fast reinem Löß entwickelt ist, während das Substrat der älteren Böden überwiegend starken Basalteinfluß aufweist. Uber die mikromorphologischen Gefügeeigenschaften von basaltisch beeinflußten Böden liegen bisher praktisch keine Untersuchungen vor. Pedogenetisch von besonderer Bedeutung ist insbesondere die große Menge des durch die Basaltverwitterung freigesetzten Tones, der sich im Dünnschliff durch fehlende Orientierung charakteristisch vom pedogenetisch verlagerten Ton abhebt.

\section{Zyklus}

Der Löß, das Ausgangssubstrat des holozänen Bodens, kann stratigraphisch wohl dem Jungwürm zugeordnet werden. Infolge der fast vollständigen holozänen Entkalkung gibt es keine direkten stratigraphischen Befunde, die diese Zuordnung bestätigen könnten. Allerdings setzen mehrere große Eiskeile innerhalb dieses Lößpaketes an. In einem dieser Eiskeile ist, da er unter die holozäne Entkalkungsgrenze hinabreicht, die einzige kalkhaltige Lößpartie des gesamten Profils erhalten. Interessant ist die am Dünnschliff gemachte Beobachtung, daß die holozäne Tonverlagerung in Grobporen sogar in das kalkhaltige Substrat hinabreicht und der Ton die mit Sekundärkalk ausgekleideten Poren überzieht, sicherlich ein Effekt, der an schnell dränende Poren gebunden ist.

Es fällt auf, daß das jungwürmzeitliche Lößpaket fast frei von Basaltmaterial ist, d. h. es besteht vor allem im oberen Abschnitt fast nur aus ferntransportiertem äolischem Material.

Zum Liegenden hin macht sich zunehmend eingespülter bzw. gelisolifluidal transportierter Basaltgrus bemerkbar. Weiterhin fällt die im Vergleich zu den älteren Lößsedimenten überdurchschnittliche Mächtigkeit der jungwürmzeitlichen Lößdecke auf. Es könnte nun gefolgert werden, daß sie tatsächlich im Vergleich zu älteren Lößablagerungen eine 
überdurchschnittliche Ablagerungsmächtigkeit besessen hat. Genausogut aber ist denkbar - und die nähere Profilanalyse bekrättigt eine solche Lösung in mehreren Fällen —, daß die älteren Lößdecken in nachfolgenden Erosionsphasen ihren oberen "rein" äolischen Abschnitt eingebüßt haben. Dafür spricht zum Beispiel, daß die älteren fossilen Böden auch nur in ihrem tiefsten Abschnitt des $B_{\mathrm{t}}$-Horizonts noch autochthon erhalten sind.

Im Liegenden der Jungwürm-Lößdecke folgt eine mächtige Basaltgelisolifluktionsdecke mit teils sehr großen unverwitterten Basaltblöcken. Diese schwimmen in einer Matrix aus Lößmaterial und Basaltgrus, der - wie im Dünnschliff zu sehen ist - teilweise starke Verwitterungsmerkmale aufweist. Diese sind zweifellos auf eine tiefgreifende Desintegration des anstehenden Basalts vor der Abtragung zurückzuführen, wie sie - abhängig wohl von der Mineralzusammensetzung der jeweiligen Basaltvarietät - verschiedentlich zu beobachten ist. Die festen Blöcke der Gelisolifluktionsdecke entstammen dann vermutlich großenteils den noch frischen Kernen, die der vom Kluftnetz ausgehenden Vergrusung noch nicht zum Opfer gefallen waren.

Unterhalb der Basaltschuttdecke folgt - nur lokal im rechten Profilteil vertreten eine krättig braune Gelisolifluktionslage aus überwiegend tonig-schluffigem Material, die in sich sehr heterogen ist; so enthält sie neben der dunkelschokoladenbraunen Grundmasse humose Schlieren, aber auch hellere, schon makroskopisch als lößbürtig zu klassifizierende Partien. Die Dünnschliffauswertung zeigt neben lößartig-schluffigen Bereichen teilweise mit Einzelkorngefüge - solche, in denen große Mengen von basaltbürtigem Ton und verwittertem Basaltgrus auftreten. Daneben kommen zerstörte und abgerollte Tonbeläge einer kräftig entwickelten Parabraunerde in großer Zahl vor. Die makroskopisch humosen Bereiche enthalten Holzkohleflitter und abgerollte Eisen-Mangan-Konkretionen. Die Körner sind frei von Eisenhüllen. Im Liegenden dieses sehr komplexen Sedimentkörpers konnten keine autochthonen Bodenreste gefunden werden. Dennoch scheint die folgende Interpretation, die mit früheren Beobachtungen RoHDENBURGs (1968) übereinstimmt, gerechtfertigt: In einer Bodenbildungszeit, die mit dem Eem-Interglazial korreliert wird, entwickelten sich verbreitet kräftige Oberflächenböden, teils Parabraunerden, teils - entsprechend dem Substrat - tonreiche Basaltverwitterungsböden. Im Frühglazial sind diese Böden und auch ein Teil der liegenden Sedimente in steileren Hangpartien einer kräftigen fluvialen Abtragungsphase zum Opfer gefallen. In flacheren Hangbereichen und auf den Basaltoberflächen müssen diese aber wenigstens zum Teil erhalten geblieben sein. Darauf erfolgte wahrscheinlich die Anwehung äolischen Materials, in dem sich nachfolgend humose Böden entwickelten. Schließlich kam es zu verstärkter Gelisolifluktion, die auch die flacheren Reliefteile und damit die dort noch erhaltenen warmzeitlichen Bodenreste erfaßte und gemeinsam mit Basaltgrus der Kuppenlagen und Lößsedimenten sowie den Humusböden verlagerte. Auch während der Phase(n) der Humusbodenbildung ist fluviale Abtragung nicht auszuschließen. Die Zone des BK 1 umfaßt gemäß dieser Deutung sowohl das echte Eem-Interglazial als auch das Frühwürm. Die Basaltgelisofluktionsdecke im Hangenden wird entsprechend ins mittlere Würm gestellt. Zu dieser Zeit müssen mindestens die Oberhänge bzw. Kuppen sedimentfrei gewesen sein, so daß die Gelisolifluktion den zwar (warmzeitlich) vergrusten, aber anstehenden Basalt angriff. Gegen das jüngere Würm - ohne daß hier eine präzise Eingrenzung möglich wäre - erlahmte infolge wachsender Aridität die Gelisolifluktion und die Lößanwehung dominierte schließlich vollkommen.

Ob der etwas erhöhte Basaltgrusanteil im allerobersten Abschnitt auf schwache jungtundrenzeitliche Reaktivierung der Gelisolifluktion oder auf holozäne Überlagerungsprozesse zurückgeht, kann mangels weiterer Befunde für diese Stelle nicht entschieden werden. 
Kurze Charakterisierung des 1. Zyklus:

Interglazialer und frühglazialer Abschnitt (Eem-Altwürm) fehlen vollständig. Das Material des interglazialen B-Horizonts sowie der Altwürm-Humuszonen ist in die durch Lößbänder gegliederte Gelisolifluktionsdecke IIIa eingearbeitet (Subtypen a3 und d3). Mittelglaziale Basaltgelisolifluktion (Subtyp d1) und jungglaziale Lößanwehung (Subtyp c2) sind durch sehr mächtige Akkumulate vertreten.

\section{2. $\mathrm{Zyklus}$}

Eine extrem stark reduzierte Serie ist die des BK 2 und seiner hangenden Sedimente. Im aufgeschlossenen Profilbereich ist offenbar nur die äußerste Zunge einer Gelisolifluktionsdecke aus Bodenmaterial entsprechend BK 1 zu erkennen. Sie lagert einer sehr geringmächtigen, aber stratigraphisch durchhaltenden Schicht auf, die aus fluvial verlagertem Basaltgrus besteht. Im Dünnschliff sind auch zahlreiche Trümmer von Tonbelägen festzustellen. Es liegt daher die Vermutung nahe, in ihr ein Zeugnis einer frühkaltzeitlichen Bodenausräumungsphase zu sehen - entsprechend den Verhältnissen im Frühwürm. Während BK 2 in seinem Habitus trotz der nur gering vorhandenen Reste deutlich die Verwandtschaft mit BK 1 erkennen läßt - z. B. lassen sich auch hier humose Partien mit verkohlten Pflanzenresten nachweisen - sind die hangenden lößbürtigen Sedimente nicht ohne weiteres zu parallelisieren. Im Dünnschliff zeigen sie gewisse Verwandtschaft mit den humosen Bereichen im BK 1. Man könnte deshalb daran denken, sie einem frühkaltzeitlichen, vielleicht auch einem mittelkaltzeitlichen Abschnitt zuzuordnen. Von allen jüngeren Sedimenten dieser Kaltzeit müßte dann angenommen werden, daß sie der frühwürmzeitlichen Abtragung zum Opfer fielen oder daß es sie nie gegeben hat. Vieles spricht durchaus für die erste Annahme, wenn man die Interpretation der geomorphologischen Entwicklung im Frühwürm akzeptiert. Diese Interpretation ist auch die Grundlage für die Zuordnung der Bodensedimente BK 1 und BK 2 zu zwei verschiedenen Warmzeiten. Die Existenz so kräftiger Ausräumungsphasen schließt die Abkunft der Gelisolifluktionsdecken BK $1 /$ BK 2 von e in em Interglazialboden praktisch aus; zudem sind sie ja deutlich durch ein lößartiges Sediment getrennt.

Kurze Charakterisierung des 2. Zyklus:

Der Zyklus ist nur in extremer Reduktion erhalten. Die Dünnschliffe lassen einen humosen Horizont (Subtyp a3) und hangende Lößsedimente (Subtyp c1) erkennen. Das Material IIIa konnte wegen inzwischen verschlechterter Aufschlußverhältnisse für die Dünnschliffanalyse nicht mehr gewonnen werden.

\section{3. $\mathrm{Zyklus}$}

Der Profilabschnitt des BK 3 sowie der überlagernden Sedimente ähnelt in seinem Aufbau recht stark dem Abschnitt des BK 2 im Hangenden. Im einzelnen ist er aber etwas vollständiger entwickelt und damit auch besser zu interpretieren. Die geringmächtige Gelisolifluktionsdecke BK 3 enthält neben (fluvial transportiertem ?) Basaltgrus viele Trümmer von Tonbelägen, die aus einem kräftigen $B_{t}$-Horizont stammen müssen. Sie entspricht damit genetisch völlig den tonigen Partien des BK 1, gehört also - in entsprechender Interpretation - einer frühglazialen Phase mit fluvialer/gelisolifluidaler Geomorphodynamik an. Im entkalkten Löß unterhalb BK 3 konnten im Dünnschliff feine Toninfiltrationslamellen aus hellgelbem Ton festgestellt werden. Es ist allerdings nicht zu entscheiden, ob diese Lamellen letzte Reste des autochthonen Bodens BK 3 oder Neubildungen einer späteren Phase darstellen, wobei prinzipiell auch das Holozän eingeschlossen sein muß. Tonlamellenbildung kann in sehr unterschiedlicher Tiefe unter der Oberfläche 
einsetzen, sofern nur genügend Sickerwasser die Stelle erreicht, der $\mathrm{pH}$-Wert im günstigen Bereich liegt und eine geeignete Tonquelle vorhanden ist. Die Interpretation der Toninfiltrationslamellen muß deshalb stets mit großer Vorsicht erfolgen. Die humosen Sedimente des oberen Abschnitt von BK 3 sind deutlich fluvial umgelagert. Ihr stark sandig-grusiger Charakter mit abgerollten Tonbelägen und Eisen-Mangan-Konkretionen zeigen dies an. Das entspricht dem Geländebefund, der Ähnlichkeiten mit einem Lehmbröckelsand vermuten ließ. Humusgehalt und verkohlte Pflanzenreste zeigen die Aquivalenz zu den frühwürmzeitlichen Humuszonen an. Insgesamt weist dieses Sediment auf einen noch frühkaltzeitlichen Abschnitt hin, in dem kurzzeitig wohl auch Parabraunerde-Dynamik nochmals aufgelebt sein kann, wie aus den frischen Tonbelägen auf Gefügekörpern des oberen Abschnitts hervorgeht. Frühkaltzeitliche Parabraunerde-Dynamik - meist allerdings wohl in bereits entkalkten Sedimenten - ist ja bereits mehrfach festgestellt worden.

Interessant ist das nach oben zunehmend stärkere Hervortreten der Lößkomponente, das wohl den Wandel zur äolischen Dominanz anzeigt. Allerdings ist festzustellen, daß die Lößsedimente oberhalb BK 3 insgesamt gelisolifluidal verlagert und mit feinem Basaltmaterial vermischt sind. Im eigentlichen Sinne „echte“ Lösse, die vielleicht mit dem Jungwürmabschnitt zu parallelisieren wären, gibt es auch in diesem Bereich nicht; sie mögen der Abtragungsphase von BK 2 zum Opfer gefallen sein.

Kurze Charasterisierung des 3. Zyklus:

Trotz geringer Schichtmächtigkeit liegt eine gut rekonstruierbare Folge vor. Die Dünnschliffe zeigen über einem Basaltverwitterungslehm (Subtyp d3) humose Bänder (Subtyp a2 und a1). Die hangenden Sedimente mit starker Schluffdominanz sind dem Mittel-Jungglazialabschnitt zuzuordnen.

\section{Zyklus}

Der BK 4 gehört zu den am besten gliederbaren Bodenkomplexen des Profils. Der eigentlich vollkaltzeitliche Abschnitt darüber ist allerdings nur schwach vertreten, und zwar in Form eines geringmächtigen Lößpaketes, dessen gelisolifluidale Verlagerung durch Basaltgruseinmengung belegt ist. Ein voll äolischer Abschnitt fehlt ebenso wie über BK 3 und BK 2, wahrscheinlich aus den gleichen Gründen.

Die Basis des Pedokomplexes ist gekennzeichnet durch nur wenig von Gelisolifluktion beeinflußte Toninfiltrationslamellen und quasi-intakte Tonbeläge in Grobporen: es sind dies Hinweise auf den tiefsten (par-) autochthonen Teil eines $\mathrm{B}_{\mathrm{t}}$-Horizonts in Löß. Darüber folgt ein braunes Bodensedimentpaket, das offenbar aus mehreren Gelisolifluktionsdecken besteht, die durch ihren teils stärkeren, teils weniger ausgeprägten Gehalt an Basaltschutt unterschieden werden können. Entsprechend ändert sich auch das Bild der Tonsubstanz, die im oberen Teil in ungeschichteter Form dominiert (Basaltverwitterung), unten aber häufig deutlich geschichtete Tontrümmer aufweist (Reste einer Lößparabraunerde). Die oberste der braunen Gelisolifluktionsdecken enthält bereits die für den Abschnitt der frühglazialen Humuszonen charakteristischen Holzkohleflitter. Insgesamt erweist sich diese Decke als sehr stark gelisolifluidal-fluvial verlagert. Das Material allerdings muß, wie auch das der liegenden Gelisolifluktionsschichten von einem interglazialen Boden (Lößparabraunerde, Basaltverwitterungsboden) abstammen.

Im Hangenden folgen mehrere humose Schichten, teilweise mit Staunässeerscheinungen über dem tonigen liegenden Gelisolifluktionsmaterial (Eisen-Mangan-Konkretionen). Streifen mit gröberer Kornsortierung lassen auf fluviale Umlagerung schließen. Holzkohle und Humusgehalt sind Beweise für die Verwandtschaft mit den Altwürm-Humuszonen. Bemerkenswert ist - wie bereits in BK 3 - die deutlich nachweisbare frühkaltzeitliche 
Tonverlagerungs-Dynamik, die durch die Existenz intakter Tonhumusbeläge nachweisbar ist. Ebenso beachtenswert ist die Einschaltung ziemlich reiner Lößzwischenschichten in die humose Serie. Das beweist erneut das Auftreten von echt kaltzeitlichen, durch äolische Dynamik geprägten Klimaphasen bereits in den frühen Abschnitten der Kaltzeiten.

Kurze Charakterisierung des 4. Zyklus:

Ein vor allem im frühglazialen Abschnitt sehr gut überlieferter Zyklus. Der interglaziale B-Horizont ist in Resten noch nachweisbar. Darüber folgt gelisolifluidal verlagerter B-Horizont (Subtyp b1). Durch dünne Lößbänder ist eine Unterbrechung markiert, nach der eine Gelisolifluktionsdecke aus Basaltverwitterungslehm (Subtyp d3) folgt. Darauf folgen die humosen Horizonte des Frühglazialabschnitts, teilweise durch dünne Lößeinschaltungen getrennt. Der untere (Subtyp a3) zeigt noch Tonverlagerung, die oberen (Subtyp a1 und a2) dagegen nicht mehr. Die hangenden Lößsedimente dürften dem Jungglazialabschnitt angehören. Es fällt auf, daß der gesamte Zyklus gegenüber anderen sehr stark durch Lößakkumulation geprägt ist.

\section{5. und 6. Z y k lus}

Bei den Sedimenten zwischen BK 4 und BK 5/BK 6 handelt es sich um lößartige Umlagerungsprodukte. Ein teilweise höherer Tongehalt zeigt die Einmischung von Basaltboden-B-Horizontmaterial an. Die Gelisolifluktion scheint bei der Umlagerung entscheidend gewesen zu sein. Im oberen Teil nimmt der Anteil an basaltischem Grobmaterial in den Gelisolifluktionsdecken stark zu. Aufgrund dieser Beobachtungen ist zu vermuten, $\mathrm{da} ß$ dieser Sedimentkomplex noch in die früheren bis mittleren Abschnitte der fünften im Profil vertretenen Kaltzeit gehört. Oberhalb des Basaltschuttes, also in dem von der Bodenbildung BK 4 überprägten Bereich, dominieren eindeutig lößartige Sedimente. In Analogie zur Würmkaltzeit könnten sie als Korrelat des kalttrockenen Milieus des jüngeren Abschnittes der Kaltzeit angesehen werden. Trotz der kräftigen pedogenen Überprägung durch BK 4 bleibt der Rückgang des Gelisolifluktionseinflusses an der Abnahme des basaltischen Materials erkennbar. Eine Serie großer Eiskeile im basalen Teil der beschriebenen Serie belegt vollkaltzeitliche Bedingungen schon während recht früher Phasen des Zyklus.

Weniger problematisch als nach den Geländebefunden zu befürchten war, ist die genetisch-stratigraphische Ansprache der B-Horizonte (besser: B-Horizontreste und ihrer Umlagerungsprodukte) BK 5 und BK 6. Der pedogenetische Befund des Geländes findet im Dünnschliff Bestätigung: Es handelt sich um eine Zone, in der mehrere Bodenbildungen einander durchdringen, mit zwischengeschalteten Verlagerungsphasen. Daß es sich dabei im engeren Sinne um warmzeitliche Bildungen handelt, kann nach den mikromorphologischen Merkmalen nicht in Zweifel gezogen werden, denn im Dünnschliff sind dicke Pakete von Tonbelägen und typische Tonanhäufungen basaltischer Verwitterungsböden dafür eindeutige Zeugnisse, sogar bei stärkeren Umlagerungserscheinungen. Auch die Mehrphasigkeit der Abfolge ist belegt. Das eigentliche Problem dieser Serie - die Zuordnung zu einem oder zu zwei Warmzeit-Kaltzeit-Zyklen - kann durch die mikromorphologischen Beobachtungen nicht gelöst werden. Autochthones Bodenmaterial bzw. B-(BC)-Horizontreste von BK 6 sind aber in den liegenden Lößsedimenten nachweisbar. Die Hauptmasse von BK 6 besteht allerdings ganz überwiegend aus durch Gelisolifluktion stark verlagertem B-Horizontmaterial mit wechselnden Basalteinmischungen. Die Lößkomponente tritt auffallend stark zurück. An der Grenze zu BK 5 tritt ein plötzlicher Substratwechsel ein: BK 5 ist in seinem Hauptteil in eindeutig lößartigem Substrat entwickelt. Daraus ist zu schließen (vgl. die Sedimentabfolge in den jüngeren Zyklen), daß zwischen BK 6 und BK 5 eine echte Kaltzeit mit einer Phase dominierender Lößanwehung im kalt-ariden Milieu entsprechend dem Jungwürm existiert haben muß. Das enge räumliche Zusammen- 
gehen von BK 5 und BK 6 ist demnach als lokale Besonderheit aufzufassen, bedingt dadurch, daß im Bereich des Aufschlusses während der sechsten Kaltzeit (bzw. an ihrem Ende) nur sehr wenig Sediment abgelagert wurde, aber andererseits während der gleichen Kaltzeit zwischen BK 6 und BK 5 auch sehr wenig abgetragen wurde (was ja für mehrere Zyklen im Bereich des Profils zutrifft). Es sei noch hinzugefügt, daß oberhalb des B-Horizonts von BK 5 eine typische frühkaltzeitliche Humuszone mit allen charakteristischen Merkmalen (Humus, Holzkohle, Kornscheidung etc.) entwickelt ist. Über BK 6 kann eine solche Zone wegen der starken pedogenen Überprägung durch BK 5 nicht erwartet werden.

Kurze Charakterisierung des 5. und 6. Zyklus:

Wegen der sehr starken Durchdringung der Bodenkomplexe 5 und 6, die sowohl die Geländeaufnahme als auch die Dünnschliffanalyse vor große Probleme stellt, sind die Zyklen hier zusammen behandelt worden. Die Interpretation der Sedimente oberhalb des BK 5 bereitet keine Schwierigkeiten. Dieser Bodenkomplex ist als gelisolifluidal-äolischer (überwiegend Subtyp c1) Abschnitt dem Mittel- und Jungglazial eines Klimazyklus zuzuordnen. Im Bodenabschnitt 5 lassen sich humose Teilabschnitte (Subtyp a2) und teilweise solche mit Tonverlagerung (Subtyp a3) feststellen. Nach einer Lößschicht (Subtyp c1) im Liegenden wiederholt sich die gleiche Abfolge (Subtyp a3 u. a2) im BK 6. Als zusätzliche Geländebefunde müssen hier noch eine Basaltsolifluktionslage oberhalb des Bodenkomplexes 6 und Reste des (par-) autochthonen B-Horizonts (Subtyp b1 und Typ b) in seinem untersten Abschnitt erwähnt werden.

\section{7. $\mathrm{Zyklus}$}

Der generelle Aufbau von BK 7 entspricht überraschend gut dem der jüngeren Zyklen, auch wenn kaum autochthone B-Horizontmerkmale erhalten sind. Der hangende Abschnitt besteht überwiegend aus Lößmaterial, das durch BK 6 pedogen überprägt, mindestens aber entkalkt ist. Der untere Teil des Lößpakets ist deutlich stärker mit Basaltgrus vermischt, so daß die Deutung als Gelisolifluktionsdecke für diesen Abschnitt voll zutrifft. Im liegenden Bereich - oberster Teil des BK 7 - findet sich der frühkaltzeitliche Abschnitt der Humuszonen in gewohnter Ausprägung wieder: Über einem gebleichten sandigen Horizont mit Holzkohle, Eisenkonkretionen und Anzeichen fluvialer Umlagerung folgt ein stärker humoser Bereich, der eine geomorphodynamisch etwas ruhigere Phase zu repräsentieren scheint. Im Dünnschliff sind die Umlagerungen deutlich nachzuweisen. Vor allem im basalen Teil ist die Zahl der aus dem Liegenden aufgenommenen $\mathrm{B}_{\mathrm{t}}$-Horizontreste (Bruchstücke von Tonbelägen) beträchtlich. Der untere Abschnitt des BK 7 besteht aus durch Gelisolifluktion stark vermischten Resten eines Basaltverwitterungsbodens, der nach der Verlagerung durch Pseudovergleyung überprägt wurde. Eine stärkere Verlagerung ist dann offenbar nicht mehr erfolgt. In dem liegenden Lößsediment sind Tonschmitzen enthalten, die (durch Gelisolifluktion verändert) den tiefsten Abschnitten des ehemaligen warmzeitlichen Bodens entstammen dürften. Insgesamt stellt BK 7 einschließlich seiner hangenden Sedimente einen recht vollständig erhaltenen Klimazyklus dar, der sich sehr gut gliedern und bis in Einzelheiten mit dem Eem-Würm-Zyklus parallelisieren läßt.

Kurze Charakterisierung des 7. Zyklus:

Der Aufbau entspricht weitgehend dem des 4. Zyklus, die Aufgliederung des frühglazialen Abschnitts ist allerdings weniger detailliert. Entsprechend zu BK 4 ist auch die starke Lößdominanz. Der interglaziale Boden ist durch einen parautochthonen $\mathrm{B}_{\mathrm{t}}$-Horizontrest (Subtyp b1) vertreten. Der hangende stärker verlagerte Abschnitt zeigt beträchtlichen Basalteinfluß (Subtyp d3). Bleichhorizont und Humuszonen entsprechen im Dünnschliff beide dem Subtyp a2. 


\section{Zyklus}

Der BK 8 ist einer der am unvollständigsten erhaltenen Komplexe. Dennoch ist seine stratigraphische Ansprache logisch befriedigend möglich. Pedogenes Material befindet sich nur in einer geringmächtigen, teils zweilagigen Gelisolifluktionsdecke. Sie enthält neben Basaltgrus und stärker verwitterten Basaltpartikeln große Mengen abgerollter und durch Gelisolifluktion zerstörter Tonbeläge, die eindeutig aus einem warmzeitlichen Boden stammen. Aquivalente zu einer Humuszone bzw. ihren Umlagerungsprodukten fehlen vollständig. Allerdings läßt die Sedimentfolge im Hangenden (zunächst Basalt-Lößgelisolifluktionsdecke mit sehr groben Basaltbrocken, dann Übergang zu reiner Lößakkumulation) keinen Zweifel darüber aufkommen, daß BK 7 und BK 8 durch eine echte, vollständige Kaltzeit getrennt sind. Es ist zu vermuten, daß das braune Bodenmaterial des BK 8 einen letzten Abtragungsrest des ehemaligen interglazialen Bodens zum Ende des frühglazialen Abschnittes darstellt, entsprechend etwa den Verhältnissen des BK 2 oder auch BK 1. Die zugehörige Humuszone wäre dann entsprechend unter BK 8 oder in ihm eingemischt zu suchen. Für eine solche Deutung kann noch angeführt werden, daß im BK 8 ein deutlich fluvial geprägtes, reichlich Basaltgrus, aber auch Bodenreste enthaltendes Sediment auftritt, das versuchsweise als Lehmbröckelsand angesprochen wurde. Bei der gegebenen Interpretation befände sich dieses Sediment in einer stratigraphisch zu erwartenden Position, wäre also konsequent dem BK 8 zuzurechnen, während es sonst einer geomorphodynamisch einsichtigen Zuordnung unzugänglich bliebe.

Kurze Charakterisierung des 8. Zyklus:

Im Bodenkomplex extrem rudimentär. Die dünne, braune (teilweise zweilagige) Gelisolifluktionsschicht enthält überwiegend Basaltmaterial; sie entspricht der Gelisolifluktion $\mathrm{zu}$ Beginn des Mittelglazials. Neuerdings konnten im Liegenden gelisolifluidal verlagerte Humuszonenreste festgestellt werden. Die mittel- und jungglaziale Abfolge zeigt den normalen Aufbau mit Basaltgelisolifluktionsdecke und Lößüberlagerung.

\section{Zyk $1 \mathrm{us}$}

Trotz seiner nur geringen Mächtigkeit hat BK 9 alle Merkmale, die man von vollständiger entwickelten jüngeren Bodenkomplexen kennt. Über einer dünnen Gelisolifluktionslage aus Bodenmaterial in normaler Ausprägung folgt ein sandig-humoser Horizont mit Eisenkonkretionen und Resten von Tonbelägen, die aus dem Liegenden aufgenommen wurden. Darüber folgt eine Lößdecke, die nicht weiter differenziert werden kann. So unergiebig an Einzelheiten der Befund auch ist, so eindeutig muß seine stratigraphische Valenz beurteilt werden: BK 9, einschließlich der hangenden Sedimente, muß als WarmzeitKaltzeit-Zyklus gerechnet werden, wobei allerdings, wie so häufig, die Phase des echten Interglazials nur indirekt, aber über widerspruchsfreie Indikatoren erschlossen werden kann.

Kurze Charakterisierung des 9. Zyklus:

Durch laminares Bodenfließen extrem ausgedünnt, aber in der ursprünglichen Schichtund Horizontabfolge erhalten. Das Dünnschliffbild zeigt zwar starke Zerstörung der Bodenmerkmale, läßt aber deutlich den ehemaligen B-Horizontbereich (Subtyp a3) und darüber den Humuszonenbereich (Subtyp a1) erkennen. Mittel- und Jungglazial sind durch lößbürtige Sedimente vertreten. Neuerdings konnten zwischen B-Horizontrest und $\mathrm{Hu}-$ mushorizontrest Fetzen vom ehemaligen Bleichhorizont festgestellt werden. 
10. $\mathrm{Zyklus}$

Die zu BK 10 gehörige kaltzeitliche Serie erscheint noch stärker reduziert. Über der allerdings sehr deutlich und zweifelsfrei ausgebildeten Humuszone, die eine Mächtigkeit von annähernd einem Meter erreicht, tritt lediglich noch eine dünne Basalt-Löß-Gelisolifluktionsdecke auf. Ahnliche Verhältnisse, wenn auch nicht mit so geringen Mächtigkeiten, waren ja bereits für jüngere Zyklen (BK 3, BK 2) festgestellt worden. Interessant ist dagegen die Ausbildung des basalen, warmzeitlichen Teiles von BK 10 in einen gelisolifluidal leicht verlagerten Pseudogley mit Vorgeschichte als Basaltverwitterungsboden, der — nach leichter Überlagerung mit sandigerem Material - erneut von einer Pseudovergleyung betroffen wurde. Interessant ist die Beobachtung, daß dieser Boden in einem Bereich, wo er in einer Lage aus feinen tertiären Quarzschottern entwickelt ist (Gelisolifluktion ?), keine Pseudovergleyung mehr aufweist und eine leuchtend braunrote Färbung annimmt. Obwohl dieser Boden durch Verlagerung in seinem Gefüge gestört ist, kann er wohl zum Parabraunerdetypus gerechnet werden.

Kurze Charakterisierung des 10. Zyklus:

Bodenkomplex gut erhalten. Der parautochthone interglaziale Bodenrest (Subtyp b2) deutet auf eine pseudovergleyte Parabraunerde hin. Im Hangenden sind zwei Humuszonen unterscheidbar (Subtyp a3 und a2), die beide im Gelände Bleichungserscheinungen erkennen lassen.

\section{1. $\mathrm{Zyk} 1 \mathrm{us}$}

Für den Bodenrest BK 11 gelten in gleicher Weise die zu BK 8, BK 2 und BK 1 gemachten Ausführungen. Mit scharfer Erosionsdiskordanz zum Liegenden treten die warmzeitlichen Bodensedimente in Form einer lebhaft schokoladenbraun gefärbten Gelisolifluktionsdecke auf, die nach oben in direktem Kontakt (und teilweise mit ihr durchdrungen) mit einer grobblockigen Basaltsolifluktionslage steht. Diese wird im Hangenden von einer fast basaltfreien Lößschicht abgelöst. Ein ehemals vorhandener Humushorizont könnte höchstens in BK 11 eingearbeitet sein. Bei der gegebenen sehr innigen Durchmischung des Gelisolifluktionsmaterials wäre er aber nur durch einen Zufallsfund, etwa von verkohlten Pflanzenresten, identifizierbar.

Bemerkenswert ist die in der Gelisolifluktionsdecke über BK 11 besonders ausgeprägte vollständige Vergrusung der Basaltblöcke. Diese kann erst nach Abschluß des Transports eingetreten sein, da die Blöcke sonst durch die Gelisolifluktionsbewegung ausgewalzt worden wären, wie dies gelegentlich schon beobachtet wurde. Welche Faktoren schließlich die Vergrusung hervorriefen, erscheint noch unklar, zumal die jüngste Basaltblockdecke des Mittelwürms aus frischem, unverwittertem Basalt besteht, die Verwitterung im Holozän also offensichtlich nicht zur Vergrusung ausreichte.

Kurze Charakterisierung des 11. Zyklus:

Es ist praktisch nur der mittel- und jungglaziale Abschnitt erhalten (vgl. Zyklus 8, aber auch 1). Im Dünnschliff läßt die Gelisolifluktionsdecke IIIa kaum Bodenmaterialanteile erkennen (ähnlich BK 8), obwohl sie im Gelände tief schokoladenbraun erscheint (Subtyp d2). Im Hangenden ist eine grobblockige Basaltgelisolifluktionsdecke entwickelt, die von fast reinem Löß überlagert wird.

\section{Zyklus}

BK 12 weist, wie alle vollständig erhaltenen, d. h. weniger durch Gelisolifluktion verlagerten Bodenkomplexe (BK 7, BK 10), deutliche Merkmale einer mehrphasigen Ent- 
wicklung auf. Wenigstens zwei Pseudogleyphasen, durch schwach umgelagertes Material getrennt, überprägen eine vorher entstandene und umgelagerte Parabraunerde. Diese schon makroskopisch festgestellte Entwicklung findet in der Dünnschliffanalyse ihre Bestätigung. Es ist einleuchtend, daß als Interglazialäquivalent im engeren Sinne nur die initiale Parabraunerde gewertet werden kann, da sich in den nachfolgenden Verlagerungsphasen bereits kaltzeitliche Bedingungen - wenn vielleicht auch nur kurzfristig - widerspiegeln. Der erste nachhaltiger wirksame Klimaeinschnitt dürfte sich allerdings erst im Übergang zum sandig-kiesigen (Tertiärgerölle ?) Sediment abzeichnen, in dem sich später ein Bleichhorizont mit Eisen-Mangan-Konkretionen ausbildete, vielleicht mit einer vorangehenden Humusbodenphase. Dieses Sediment, dessen Aquivalente ja in verschiedenen Klimazyklen durchaus flächige Verbreitung zu besitzen scheinen, ist eindeutig fluvialen Ursprungs. Es ist vielleicht nicht falsch, in der Zeit seiner Ablagerung oder kurz vorher anderenorts flächige Ausräumung der Oberflächenböden bzw. oberflächennahen Sedimente $\mathrm{zu}$ vermuten, wie sie ja in anderen Zyklen des Profils zu fordern ist. Es fällt auf, daß kräftige Bleichhorizonte nur für gut erhaltene Bodenkomplexe typisch sind (BK 5, BK 7, BK 12), d. h. für solche, die sich im Frühglazial in Bereichen geringer Abtragung bis Akkumulation befanden, denn sicherlich ist die Ausbildung stark entwickelter Bleichhorizonte substratspezifisch. Wo sandige Sedimente abgelagert wurden, war ihre Entwicklung bevorzugt. Oberhalb des Bleichhorizonts folgt, wie auch schon in BK 7 beschrieben, eine humose Zone, die ebenfalls aus stärker sandigen (fluvialen!) Sedimenten besteht, aber insgesamt eher schon ein Ausklingen der starken fluvialen Prozesse signalisiert. Die oberhalb BK 12 folgenden Basalt-Löß-Gelisolifluktionsdecke ist sicher, wie bereits erwähnt, nur als Abtragungsrest (Phase III) einer ehemals mächtigeren kaltzeitlichen Serie anzusehen.

Kurze Charakterisierung des 12. Zyklus:

Der fast fehlende Basalteinfluß fällt ins Auge. Stattdessen ist vor allem im fluvialen Abschnitt ein hoher Sandanteil (Tertiärsande der Umgebung) zu vermerken (Subtypen a4 und b3). Im übrigen starke Ähnlichkeit im Aufbau mit Zyklus 10. Besonders die kräftige Pseudovergleyung am Beginn des Frühglazials ist beiden gemeinsam. Mittel- und Jungglazialabschnitt sind in üblicher Form vertreten.

\section{3. $\mathrm{Zy} \mathrm{klus}$}

Der letzte im Profil stratigraphisch klar greifbare Bodenrest BK 13 stellt wieder nur ein rudimentäres Umlagerungsprodukt dar, wie es auch in BK 11, 8, 3, 2 und 1 auftritt. Dennoch bestehen über seine Eigenart keine Zweifel. Das Auftreten von Holzkohleflittern und zerstörten Tonbelägen erlaubt eine klare Ansprache. Zudem ist BK 13 in charakteristischer, bereits bekannter Weise mit groben Basaltsolifluktionsschutt vergesellschaftet, der im Hangenden von lößartigen Sedimenten bedeckt wird, die allerdings im Gegensatz zu jüngeren Bildungen sehr sandreich sind. Unterhalb BK 13 folgen Sande, feine Schotter und Tone tertiärer Herkunft, unter denen im klüftigen, teils zersetzten Basalt Reste eines weiteren Bodens gefunden werden können, für den aber eindeutige Zuordnungen nicht mehr möglich sind.

Kurze Charakterisierung des 13. Zyklus:

Im Bodenkomplex sehr unvollständig (Subtyp b3). Die Verzahnung mit einer Basaltgelisolifluktionsdecke legt die Einstufung in die Phase III a nahe. Der Aufbau entspricht somit den Zyklen 11 und 8. Die (jungglazialen) hangenden Lößsedimente sind sehr stark mit Sand vermischt. 


\section{Schlußbetrachtungen}

In allen Zyklen treten - bei generalisierender Betrachtung - im wesentlichen nur 5 Sedimenttypen auf:

1. Schwach solifluidal umgelagerte Reste warmzeitlicher Böden;

2. überwiegend fluviale Sedimente mit nachfolgender Bleichung, oder humos;

3. sehr stark gelisolifluidal verlagertes Bodenmaterial;

4. Basalt-Löß-Gelisolifluktionsdecken;

5. Lößsedimente mit relativ geringem Basaltanteil.

Diese Sedimenttypen treten nicht in regelloser Folge auf, sondern - wo sie nicht fehlen - stets in der angegebenen Reihung. Das läßt es gerechtfertigt erscheinen, ihr Fehlen zunächst als lokale Besonderheit aufzufassen, in ihrer Reihung aber regelhafte Prozeßabläufe zu vermuten, die für alle oder doch viele Klimazyklen des Quartärs in gleicher Weise charakteristisch sind. Zu ähnlichen Schlüssen ist schon KuKL.A (1969) aufgrund der Analyse tschechischer Lößprofile gekommen, wobei ihm der hohe Detaillierungsgrad dieser Profile weitgehende Schlußfolgerungen erlaubte. Doch auch für das im Detail weniger differenzierte Profil Dreihausen kann das Modell eines idealen Klimazyklus entworfen werden, dessen überregionale Bedeutung sicherlich im einzelnen zu überprüfen ist.

Nach den vorliegenden Befunden hätte man im Verlauf eines Warmzeit-KaltzeitZyklus mit folgender generellen Entwicklung zu rechnen:

Im Interglazial intensive Bodenentwicklung (Parabraunerde aus Löß, tonreiche Verwitterungsböden aus Basaltzersatz).

Im Übergang zum Frühglazial kommt es kurzfristig zu gelisolifluidalen Bodenbewegungen. Insgesamt aber überwiegt weiterhin die Pedogenese. Je nach Standort kommt es zur Tonverlagerung oder zur Pseudovergleyung. Es können mehrere kurzzeitige Unterbrechungen der Pedogenese, verknüptt mit relativ schwachen Bodenbewegungen, auftreten.

Eintritt einer ersten krä̈tigen, vorwiegend fluvialen Abtragungsphase. Diese Phase muß mit einem erheblichen Umbruch in der Vegetation verknüptt gewesen sein. Oberflächenböden werden zumindest teilweise abgeräumt. Gegen Ende dieser Phase stellenweise Akkumulation eines sandigen Sediments und Bildung eines Bleichhorizonts mit Eisen-Manganausfällungen. Nach erneuter Abtragung und Sedimentation bilden sich humose Böden, oder es werden vorher gebildete humose Sedimente verlagert und akkumuliert. Kurzzeitig tritt in kalkfreien Substraten noch Tonverlagerung auf, in nachfolgenden Unterbrechungsphasen der Bildung humoser Böden kommt es bereits kurzzeitig zu kräftigerer Gelisolifluktion und auch geringer Lößanwehung (Lößbeimengung in oberen Partien der humosen Zonen).

Im Übergang zum mittleren Glazialabschnitt tritt kräftige Gelisolifluktion ein, die zunächst bevorzugt tonigere Substrate (also noch vorhandene Bodenreste) betrifft; schließlich wird aber auch grobes Basaltmaterial und Löß transportiert. Im jüngeren Glazialabschnitt tritt die Gelisolifluktion zurück und die Ablagerung lößbürtiger Sedimente dominiert, aus denen sich mit Beginn des folgenden Interglazials eine neue Parabraunerde entwickelt.

Die Prüfung, ob dieses einfache Modell weitere Bedeutung hat, kann für den EemWürm-Zyklus sehr schnell getroffen werden, da unsere Kenntnisse hinreichend detailliert sind (ROHDENBURG 1972: 299). Auch für die vorangehende Kaltzeit hat Bibus (1974) ein entsprechendes Gliederungsschema vorgelegt. Für alle älteren Zyklen gibt es aber so gut wie keine Vergleichsmöglichkeiten zur Überprüfung (s. aber KukLA 1969). Es bleibt die Frage, ob tatsächlich alle Klimazyklen so gleichartig waren, wie es in dem entwickelten 
Modell als Arbeitshypothese vorgeschlagen wird. Dazu kann im Augenblick nur wiederholt werden, was wir bereits früher feststellten: alle im Profil Dreihausen auftretende Zyklen weisen herkömmlich als vollkaltzeitlich gewertete Abschnitte auf (starke Gelisolifluktion, Lößanwehung, z. T. Eiskeile). In den Bodenresten dagegen könnte sich von den jüngeren zu den älteren eine Differenzierung widerspiegeln, denn in den älteren treten vermehrt rote Eisenausscheidungsformen auf, die als charakteristisch für wärmere Klimate gelten. Selbstverständlich könnte sich auch die Gewichtung einzelner Phasen innerhalb der Klimazyklen geändert haben, ohne daß dies in den Profilen sichtbar würde.

Der Grund für die sehr ausführliche Bearbeitung des Profils Dreihausen liegt in seiner prinzipiellen Bedeutung für unsere Vorstellung über die Gliederung des Quartärs. Profile mit ähnlich hoher Zahl quartärer Klimazyklen sind bisher nur sehr wenige bekannt geworden, nur in einem Fall (Kukla 1969) wurde eine ins Detail gehende Analyse der stratigraphischen Befunde versucht. Ein Hauptproblem liegt in dem Mangel an Korrelierbarkeit mit unvollständigeren Serien. Dieser Mangel kann nur durch geeignete Zeitmarken behoben werden. Als solche bieten sich derzeit insbesondere paläomagnetische Daten an. Die am Profil Dreihausen von Herrn Dipl.-Phys. Fromm begonnenen paläomagnetischen Untersuchungen haben leider bisher noch nicht zu verwertbaren Ergebnissen geführt. Der schon anfangs als besonders problematisch bezeichnete starke Einfluß der Gelisolifluktion im Profil Dreihausen mit der Konsequenz einer starken Durchmischung der Sedimente mit Basalt hat gerade für die paläomagnetischen Messungen große Nachteile, denn die Proben müssen sehr stark abmagnetisiert werden und der für die Messung verbleibende Magnetismus wird dadurch sehr schwach. Da paläomagnetische Daten zur Zeit wohl die einzigen allgemein anerkannten Überprüfungskriterien für die am Quartärprofil Dreihausen aufgestellten Hypothesen über den Verlauf der quartären Klimaschwankungen darstellen, ist nur zu hoffen, daß die im Gange befindlichen Untersuchungen noch zu klärenden Ergebnissen führen.

Die Deutsche Forschungsgemeinschaft stellte von 1974 bis 1975 für ergänzende Profiluntersuchungen, die Entnahme zahlreicher Proben und für deren mikromorphologische Analyse Personalund Sachmittel zur Verfügung. Für die großzügige Förderung der Untersuchungen sprechen die Autoren der DFG ihren aufrichtigen Dank aus.

Die Fa. Nickel unterstützte die Geländearbeiten tatkräftig und ermöglichte während vieler Monate die ungestörte Untersuchung des Profils, weil sie den Abbau an dieser Stelle ruhen ließ. Für dieses Entgegenkommen und die vielfältige Hilfe sei herzlich gedankt.

Den Technischen Angestellten Hugo Althen, Adam Lapp, Walter Will und Reinhold Will danken wir für die schwierige Entnahme von Bodenmonolithen und Rahmenproben.

\section{Schriftenverzeichnis}

BıBus, E. (1974): Abtragungs- und Bodenbildungsphasen im Rißlöß. - Eiszeitalter u. Gegenwart, 25: 166-182; OOhringen/Württ.

Boenigk, W., Heye, D., Schirmer, W. \& Brunnacker, K. (1974): Paläomagnetische Messungen an vielgliedrigen Quartärprofilen. - Mz. naturwiss. Arch., 12: 159-168; Mainz.

Frenzel, B. (1973): 3. On the Pleistocene Vegetation History. - In: Schönhals, E. \& HuckRIEDE, R. (eds.): State of Research on the Quaternary of the Federal Republic of Germany: C. Area between the Scandinavian and the Alpine Glaciation. - Eiszeitalter u. Gegenwart, 23/24: 321-332; Öhringen/Württ.

Koči, A., Schirmer, W. \& Brunnacker, K. (1973): Paläomagnetische Daten aus dem mittleren Pleistozän des Rhein-Main-Raumes. - N. Jb. Geol. Paläont., Mh., 9: 545-554; Stuttgart.

KuKLA, J. (1969): Die Zyklische Entwicklung und absolute Datierung der Lößserien. - In: Demek, J. \& Kukla, J. (eds.): Periglazialzone, Löß und Paläolithikum der Tschechoslowakei: 75-95; Brno. 
LožEK, V. (1966): Die quartäre Klimaentwicklung in der Tschechoslowakei. Quartär, 17: 1-19; Bonn.

Menke, B. \& Behre, K.-E. (1973): History of Vegetation and Biostratigraphy. - In: Schönhals, E. \& Huckriede, R. (eds.): State of Research on the Quaternary of the Federal Republic of Germany: A. Area of the Scandinavian Glaciation. - Eiszeitalter u. Gegenwart, 23/24: 251-267; OOhringen/Württ.

Rondenburg, H. (1968): Jungpleistozäne Hangformung in Mitteleuropa. Beiträge zur Kenntnis, Deutung und Bedeutung ihrer räumlichen und zeitlichen Differenzierung. - Göttinger Bodenkdl. Ber., 6: 3-107; Göttingen.

- (1971): Einführung in die klimagenetische Geomorphologie. - 350 S., 39 Abb., 2 Bilder, 2 Tab.; Gießen.

- \& SABELBERG, U. (1973): Quartäre Klimazyklen im Westlichen Mediterrangebiet und ihre Auswirkungen auf die Relief- und Bodenentwicklung. - Catena, 1: 71-180; Gießen.

Sabelberg, U., Rohdenburg, H. \& Havelberg, G. (1974): Bodenstratigraphische und geomorphologische Untersuchungen an den Lößprofilen Ostheim (Kreis Hanau) und Dreihausen (Kreis Marburg) und ihre Bedeutung für die Gliederung des Quartärs in Mitteleuropa. - RheinMain-Forsch., 18: 101-120; Frankfurt a. M.

- \& Rohdenburg, H. (1975): Stratigraphische Stellung und klimatisch-geologischer Aussagewert der Kalkkrusten in Spanien und Marokko. - In: Vogt, T. (Hrsg.): Comptes-rendus du Colloque „Types de croûtes calcaires et leur répartition régionale“: 120-127, Strasbourg.

Semmel, A. (1967 a): Über Prä-Würm-Lösse in Hessen. - Notizbl. hess. L.-Amt Bodenforsch., 95: 239-241; Wiesbaden (1967 b): Das Lößprofil der Ziegelei Grün in Reinheim: Nachr. naturwiss. Ver. Aschaffenburg, 74: 108-112; Aschaffenburg.

- (1972): Fragen der Quartärstratigraphie im Mittel- und Oberrheingebiet. - Jber. u. Mitt. oberrhein. geol. Ver., 54: 61-71; Stuttgart.

- (1973): Periglacial Sediments and their Stratigraphy. - In: Schönhals, E. \& Huckriede, R. (eds.): State of Research on the Quaternary of the Federal Republic of Germany: C. Area between the Scandinavian an the Alpine Glaciation. - Eiszeitalter u. Gegenwart, 23/24: 293-305; O'hringen/Württ.

- (1974): Der Stand der Eiszeitforschung im Rhein-Main-Gebiet. - Rhein-Main. Forsch., 78: 9-56; Frankfurt a. M. 